\title{
Two NRAMP6 Isoforms Function as Iron and Manganese Transporters and Contribute to Disease Resistance in Rice
}

\author{
Cristina Peris-Peris, ${ }^{1}$ Albert Serra-Cardona, ${ }^{2}$ Ferrán Sánchez-Sanuy, ${ }^{1}$ Sonia Campo, ${ }^{1}$ Joaquin Ariño, ${ }^{2}$ \\ and Blanca San Segundo ${ }^{1}$
}

${ }^{1}$ Centre for Research in Agricultural Genomics (CRAG) CSIC-IRTA-UAB-UB. Edifici CRAG, Campus UAB, Bellaterra
(Cerdanyola del Vallés), 08193 Barcelona, Spain; and ${ }^{2}$ Institut de Biotecnologia i Biomedicina and Departament de
Bioquímica i Biologia Molecular, Universitat Autònoma de Barcelona, 08193, Cerdanyola del Vallès, Barcelona, Spain

Accepted 6 March 2017.

\begin{abstract}
Metal ions are essential elements for all living organisms. However, metals can be toxic when present in excess. In plants, metal homeostasis is partly achieved through the function of metal transporters, including the diverse natural resistance-associated macrophage proteins (NRAMP). Among them, the OsNramp6 gene encodes a previously uncharacterized member of the rice NRAMP family that undergoes alternative splicing to produce different NRAMP6 proteins. In this work, we determined the metal transport activity and biological role of the full-length and the shortest NRAMP6 proteins (l-NRAMP6 and s-NRAMP6, respectively). Both I-NRAMP6 and s-NRAMP6 are plasma membrane-localized proteins that function as iron and manganese transporters. The expression of l-Nramp6 and s-Nramp6 is regulated during infection with the fungal pathogen Magnaporthe oryzae, albeit with different kinetics. Rice plants grown under high iron supply show stronger induction of rice defense genes and enhanced resistance to M. oryzae infection. Also, loss of function of OsNramp6 results in enhanced resistance to M. oryzae, supporting the idea that OsNramp6 negatively regulates rice immunity. Furthermore, nramp6 plants showed reduced biomass, pointing to a role of OsNramp6 in plant growth. A better understanding of OsNramp6-mediated mechanisms underlying disease resistance in rice will help in developing appropriate strategies for crop protection.
\end{abstract}

Mineral nutrients are essential for normal plant growth and development. Some metals are required in trace amounts as essential micronutrients, such as iron $(\mathrm{Fe})$, copper $(\mathrm{Cu})$, manganese $(\mathrm{Mn})$, and zinc $(\mathrm{Zn})$ that serve structural roles in proteins or function as enzyme cofactors. They are also components of cellular redox reactions (Hall and Williams 2003). However, if present in excess in a bioavailable form, these essential micronutrients can be harmful to cells through the generation of toxic reactive oxygen species (ROS) or by replacement of other metal

Current address of A. Serra-Cardona: Institute for Cancer Genetics, Columbia University Medical Center, New York 10032.

Corresponding author: B. San Segundo;

E-mail: blanca.sansegundo@cragenomica.es

*The $\boldsymbol{e}$-Xtra logo stands for "electronic extra" and indicates that one supplementary table and five supplementary figures are published online.

(C) 2017 The American Phytopathological Society ions from metalloproteins, rendering these proteins nonfunctional. On the other hand, if plants do not have a sufficient supply of these metals, deficiency symptoms develop (e.g., chlorosis, necrotic spots, loss of leaves, or stunted growth). Therefore, plants have developed finely tuned homeostatic mechanisms to ensure the appropriate concentrations of essential metals at the cellular and the whole-plant levels.

Diverse protein families are known to be components of the metal homeostatic network in plants, such as the Zn and Fe ZIP (Zrt- and Irt-related protein) transporters, the natural resistanceassociated macrophage protein (NRAMP) family of divalent metal transporters, the copper transporter $\mathrm{Cu}$ transporters, the $\mathrm{Cu}$-transporting P-type ATPases, the ferric-chelate reductases, and the yellow stripe-like (YSL) transporters (Curie and Briat 2003; de Carvalho Victoria et al. 2012; Guerinot 2000; Kobayashi and Nishizawa 2012; Puig and Peñarrubia 2009; Thomine and Vert 2013; Thomine et al. 2000; Williams and Pittman 2010). Transporters localized at the plant cell plasma membrane control metal ion uptake and release, whereas those localized in endogenous subcellular compartments are mainly responsible for sequestration and remobilization of metal ions in the cell. Metal transporters are also involved in detoxification of heavy metals such as cadmium (Cd) or arsenic (As) (Cailliatte et al. 2009; Thomine et al. 2000; Tiwari et al. 2014).

The NRAMP proteins were first identified in rat macrophages (Vidal et al. 1993) and, today, they are recognized as a ubiquitous family of metal transporters present in fungi, animals, plants, and bacteria (Cellier et al. 1996, 1995; Nelson 1999). NRAMP are integral, membrane-spanning proteins usually encoded by large gene families in plants, indicating a possible functional specialization. Moreover, plant NRAMP proteins complement yeast mutants deficient in metal uptake (e.g., Fe or Mn transport), demonstrating their conserved function as metal transporters across all kingdoms (Curie et al. 2000; Gross et al. 2003; Thomine et al. 2000; Wu et al. 2016). Although they differ in their specificity, members of the NRAMP family are capable of transporting divalent metal cations $\left(\mathrm{Fe}^{2+}, \mathrm{Mn}^{2+}\right.$, $\mathrm{Zn}^{2+}, \mathrm{Cd}^{2+}$, and $\mathrm{As}^{2+}$ ) into the cytoplasm (Nevo and Nelson 2006), with the exception of the rice NRAMP aluminum (Al) transporter 1 (Nrat1) (OsNRAMP4) protein, which transports the trivalent $\mathrm{Al}$ ion $\left(\mathrm{Al}^{3+}\right)$ ( $\mathrm{Li}$ et al. 2014). Plant NRAMP proteins localize at different subcellular compartments, including the plasma membrane, tonoplast, vesicles, or plastid envelope (Cailliatte et al. 2010, 2009; Lanquar et al. 2005; Sasaki et al. 2012; Takahashi et al. 2011; Thomine et al. 2003; Xiao et al. 2008; Yang et al. 2013). 
The function of certain NRAMP in controlling plant growth and development has been elucidated, mostly in the model plant Arabidopsis thaliana. For instance, AtNRAMP3 and AtNRAMP4 contribute to Fe nutrition in germinating seed by remobilizing vacuolar Fe stores (Curie et al. 2000; Lanquar et al. 2005; Thomine et al. 2000, 2003) and to the export of vacuolar Mn in photosynthetic tissues of adult Arabidopsis plants (Lanquar et al. 2010). The NRAMP3 protein also contributes to $\mathrm{Cd}^{2+}$ sensitivity in Arabidopsis (Thomine et al. 2000). AtNRAMP1 appears to be essential for Arabidopsis growth in low-Mn conditions (Cailliatte et al. 2010) whereas AtNRAMP6 functions as an intracellular Cd transporter that increases $\mathrm{Cd}$ sensitivity when overexpressed in Arabidopsis (Cailliatte et al. 2009).

Several studies demonstrated a pathogen-regulated expression of Nramp genes in different plant species, including rice and Arabidopsis (Segond et al. 2009; Zhou and Yang 2004). However, the biological role of NRAMP during plant-microbe interactions and disease resistance is still obscure. Work carried out in the model system of Arabidopsis revealed that AtNRAMP3 and AtNRAMP4 regulate basal resistance to the bacterial pathogen Erwinia chrysanthemi (syn. Dickeya dadantii) (Segond et al. 2009). In other studies, MtNrampl was shown to function in rhizobia-infected nodule cells in Medicago truncatula (TejadaJiménez et al. 2015). These pieces of evidence support the idea that Nramp genes might be important players in plant-microbe interactions but the exact mechanisms by which NRAMP proteins and metal homeostasis may control plant immunity remain unclear.

Metals are essential not only for plant growth but also for the expression of pathogenicity and virulence genes (Fones and Preston 2012; Lemanceau et al. 2009). Accordingly, pathogens have developed sophisticated strategies for metal acquisition during growth in plant tissues, including the production of multiple high-affinity Fe-chelating compounds, or siderophores (Dellagi et al. 2009).

Rice (Oryza sativa L) is one of the most important cereal crops in the world and the main staple food crop for more than $50 \%$ of the world's population. The rice genome contains eight genes encoding NRAMP proteins (OsNrampl to OsNramp8) (Belouchi et al. 1997; Gross et al. 2003). Among them, OsNRAMP1 was reported to transport $\mathrm{Fe}, \mathrm{Cd}$, and As but not Mn (Curie et al. 2000; Takahashi et al. 2011; Tiwari et al. 2014), whereas OsNRAMP3 functions in Mn transport but not in $\mathrm{Fe}$ or $\mathrm{Cd}$ transport (Yang et al. 2013). OsNRAMP5 is an Fe, Mn, and Cd transporter (Ishimaru et al. 2012; Sasaki et al. 2012). Rice OsNRAMP4 (also known as Nrat1) was identified as a transporter for Al that does not show transport activity for divalent cations in yeast (Xia et al. 2010). No information is currently available on the metal transport capabilities or the biological role of other NRAMP family members in rice.

In this study, we approached the functional characterization of OsNRAMP6, a member of the rice NRAMP family, in the context of disease resistance. In particular, we investigated the role of OsNramp6 during interaction of rice plants with the fungal pathogen Magnaporthe oryzae. This fungus is the causal agent of the rice blast disease, one of the most devastating diseases of cultivated rice due to its widespread distribution and destructiveness (Wilson and Talbot 2009). It is also known that $M$. oryzae produces siderophores to ensure a sufficient supply of Fe during host colonization (Hof et al. 2009; Lemanceau et al. 2009). Owing to its scientific and economic relevance, rice blast is considered the most important fungal disease in plants (Dean et al. 2012).

Gene OsNramp6 presents a complex transcriptional pattern and produces up to eight transcript variants by alternative splicing encoding OsNRAMP6 proteins of different sizes (Campo et al. 2013). In a previous study focusing on the identification of micro (mi)RNA that are regulated by treatment with $M$. oryzae elicitors, we reported that miR7695, a previously uncharacterized rice miRNA, negatively regulates the expression of the shortest transcript variant of Nramp6 (Campo et al. 2013). Moreover, miR7695 overexpression confers resistance to M. oryzae infection (Campo et al. 2013). In this study, we show that both the full-length and the shortest OsNRAMP6 proteins localize at the plasma membrane. Functional complementation of yeast mutants revealed that the two NRAMP6 protein isoforms function as $\mathrm{Fe}$ and $\mathrm{Mn}$ transporters. We also provide evidence that loss-of-function of OsNramp6 confers enhanced resistance to infection by the rice blast fungus $M$. oryzae. Fe supply affects defense gene expression and resistance to the rice blast fungus $M$. oryzae. Collectively, our results support a role for OsNramp6 in rice immunity against $M$. oryzae.

\section{RESULTS}

The OsNramp6 gene primarily is known to produce up to eight transcript isoforms: the full-length transcript corresponds to the longest transcript variant (l-Nramp6, Os01g31870.1), whereas the shortest ( $s$-Nramp6, Os01g31870.8) is generated by miss-splicing of intron 6 (Fig. 1A). Reverse transcription-quantitative polymerase chain reaction (RT-qPCR) using transcript-specific primers revealed accumulation of both l-Nramp6 (full-length) and s-Nramp6 (alternatively spliced) transcripts in leaves of rice plants at the different developmental stages $(7,14,21$, and 28 days), thus supporting coexistence of both transcript isoforms in this tissue during development (Fig. 1B). The l-Nramp6 transcripts accumulated at much higher levels than $s$-Nramp6 transcripts (Fig. 1B).

The protein encoded by the l-Nramp6 transcript is 550 amino acids long whereas that encoded by the $s$-Nramp6 transcript is 210 amino acids in length (corresponding to amino acids 1 to 203 of the full-length protein plus a C-terminal extension of 7 amino acids) (Fig. 1A and C). SOSUI transmembrane (TM) domain searches (Hirokawa et al. 1998) predicted nine TM domains in 1-NRAMP6 (Fig. 1C). By using the same prediction system for other rice NRAMP, 10 (NRAMP2 and NRAMP5) and 11 (NRAMP1, NRAMP3, NRAMP4, and NRAMP7) TM domains are predicted (data not shown). In the case of s-NRAMP6, only three TM domains are predicted (Fig. 1C), as is also the case for the protein deduced from the OsNramp 8 cDNA sequence (Supplementary Fig. S1). The consensus transport motif (GQSSTITG TYAGQFIMGGFLN), which is common among plant NRAMP proteins, is only partially conserved in l-NRAMP6, and it is absent in s-NRAMP6 (Fig. 1C). Finally, NRAMP6 shares the highest amino acid sequence identity with OsNRAMP5 and OsNRAMP1 (51.3 and $48.7 \%$, respectively), OsNRAMP4 (44.8\%), and OsNRAMP3 (42.0\%), with lesser identity with OsNRAMP2 (28.5\%) and OsNRAMP7 (26.6\%).

\section{Predicted tridimensional structure of OsNRAMP6.}

The crystal-structure of Staphylococcus capitis DMT (ScaDMT), a prokaryotic homolog of the NRAMP family also known as Solute Carrier 11 (SLC11), has been recently determined (Ehrnstorfer et al. 2014). The ScaDMT structure defines the common architecture of the NRAMP family and contains 11 TM helices forming two related halves interrupted by short loops, of which the first five $\alpha$-helices are structurally related to the following five $\alpha$-helices. The Asp (D) and Asn (N) residues at the metal-binding site are part of a conserved DPGN motif identified as a signature for the family. ScaDMT transports $\mathrm{Mn}^{2+}, \mathrm{Fe}^{2+}$, and $\mathrm{Cd}^{2+}$, and mutations in metal binding residues (D49, N52, A223, and M226) have important effects on metal affinity or selectivity of this transporter (Ehrnstorfer et al. 2014). The NRAMP6 protein shares $28.34 \%$ identity with the ScaDMT protein (Supplementary Fig. S2). 
The ScaDMT protein (Ehrnstorfer et al. 2014) was used for structure modeling of the NRAMP6 protein. The tridimensional structure of OsNRAMP6 showed a high similarity to the ScaDMT structure, the largest differences being observed in the $\mathrm{N}$ and $\mathrm{C}$ terminus (Fig. 2A). We determined the ion binding residues based on positional homology to ScaDMT: Asp88 and Asn91 in NRAMP6 correspond to Asp49 and Asn52 in ScaDMT (Fig. 2B), and Ser260 and Val263 in NRAMP6 correspond to Ala223 and Met226 (Fig. 2B).

OSNRAMP6 is a plasma membrane protein.

To investigate the subcellular localization of NRAMP6 in the plant cell, we transiently expressed an OsNRAMP6-green fluorescent protein $(G F P)$ fusion gene in Nicotiana benthamiana leaf epidermal cells (Fig. 3A). The subcellular localization of the 1-NRAMP6 and s-NRAMP6 proteins was examined by confocal laser-scanning microscopy (CLSM).

Cells expressing the l-NRAMP6-GFP gene revealed a continuous labeling all along the cell periphery, likely the plasma membrane (Fig. 3B). Discrete regions enriched in GFP fluorescence embedded in this subcellular compartment as well as vesicles exhibiting GFP fluorescence located near the cell periphery were occasionally observed (Fig. 3B, arrows). A similar localization was observed in $N$. benthamiana cells expressing the $s$-NRAMP6-GFP gene (Fig. 3C). However, in this case, the pattern of fluorescence was discontinuous along the cell periphery, with random distribution of discrete domains accumulating strong fluorescence (Fig. 3C, right panel). GFP-expressing control cells showed a distribution of green fluorescence through the cytoplasm and the nucleus (Fig. 3D).

To confirm the plasma membrane localization of the NRAMP6 protein, each GFP-tagged NRAMP gene was coexpressed with the plasma membrane marker LOW-TEMPERATURE-INDUCED6b ( $L T I 6 b)$ fused to the red fluorescent protein $(R F P-L T I 6 b)$ (Kurup et al. 2005). The continuous pattern of GFP fluorescence observed with the 1-NRAMP6 perfectly colocalized with the RFP-tagged LTIB6b protein, thus confirming its plasma membrane localization (Fig. 3E). Again, the colocalization was uneven in cells
A

I-Nramp6 (Os01g31870.1)

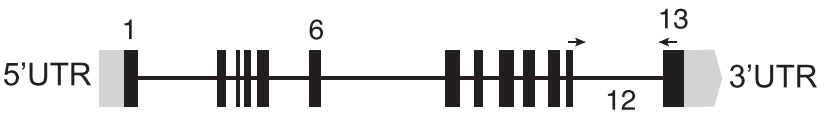

s-Nramp6 (Os01g31870.8)

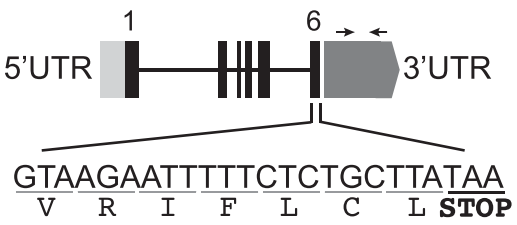

B

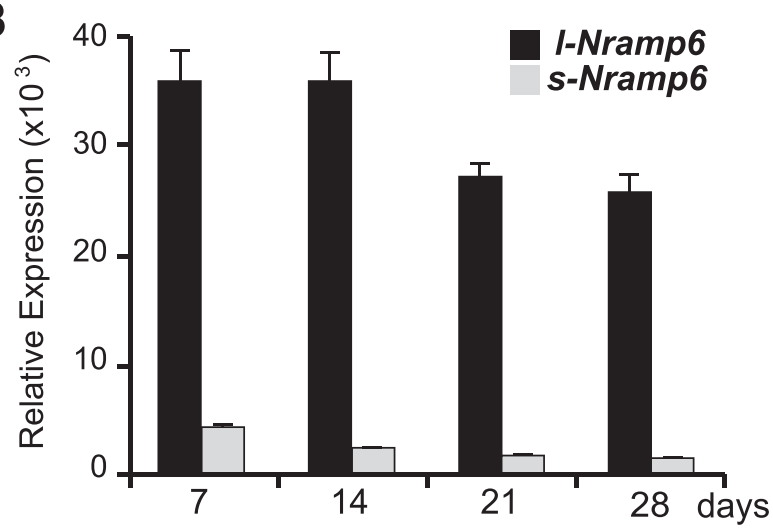

C

I-NRAMP6 MEEGAK I GREHE $Q Q Q Q$ HGRVNGSGRVAAVGGGSGGGGDE IEI EVAAAAGASPSRQHGGLHGDVQAPTWKRF LAHVGPGF s-NRAMP6 MEEGAK I GREH EQQQQQHGRVNGS GRVAAVGG GS GGG GDE I I I EVAAAA GAS P SR QH GGLHGDVQAP TWKRF LAHVGP GF

I-NRAMP6 VISIAYLDPSNLQTDLVAGSSHRYSLLWVLLFGF IFVLTVQSLAANLG I ITGRHLAELCMGEYPKYVKYCLWLLAELGVI s-NRAMP6 VIS I AYLDP SNLQT DLVAGS SHRY S LLWVLLF GF I FVLTVQSLAANLG I I TGRHLAELCMGEYPKYVKYC LWLLAELGVI

S-NRAMP6 VIS IAYLDPSNLQTDLVAGSSHRYSLLWVLLF GF IFVLTVQSLAANLGIITGRHLAELCWCI IV

I-NRAMP6 $\overline{\text { AATIPGVLGTALAYNMLLHI PFWAGVLACGACTFLILGLQ GYGARKMEFTISVLMLVMATCFFMELGKVNPPAGGVIEGL }}$ S-NRAMP6 AAT I P GVLGTALAYNMLLH I P FWAGVLAC GAC T F L I L G LQG Y GVR I F L CL L

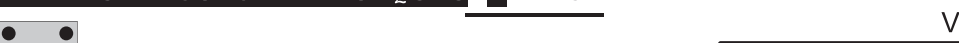

I-NRAMP6 FIPRPKGDYSTSDAVAMFGSLVVPHNLFLHSSLVLTRKMPYTSKGRKDASTFFLLENALALF IALLVNVA IVS I SGT I CA

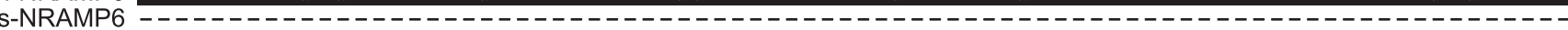

I-NRAMP6 NNLSFADTSTCSSLTLNSTYVLLKN I LGKS S STVYGVALLVSGQSCMVATSYAGQY IMQGF SGMRKC I I YLVAPCFTLLP

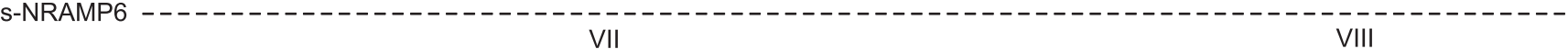
I-NRAMP6 $\overline{\text { SLI I CSIGGTLRVHR IINIAAIVLSFVLPFALIPLIKFSSSCTNI GPYKNATSIIRIAWILSLVIIGINIYFFCTSFVAW }}$ S-NRAMP6 -

IX

I-NRAMP6 LVHSDLPRVVNAI I SS LVF PFMAAY IAALIYLAFRKVNLSDPFPTNSVSGEIEVQH I QIQEKQEDLGVHL

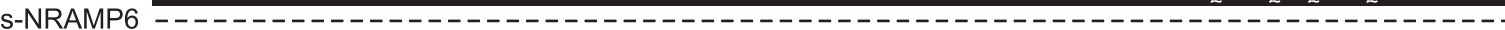

Fig. 1. Gene structure, OsNramp6 expression, and amino acid sequences of OsNRAMP6. A, Schematic representation of the OsNramp6 gene. Black bars and lines represent exons and introns, respectively. Numbers indicate the first and last exon of each l-Nramp6 and $s$-Nramp6 transcript. The long Nramp6 (l-Nramp6, Os01g31870.1) transcript encodes the full-length NRAMP6 protein (amino acids 1 to 550) and was taken as reference for intron or exon numbering. Miss-splicing of intron 6 produces a short Nramp6 (s-Nramp6, Os01g31870.8) transcript that introduces a stop codon 21 nucleotides downstream from the Exon6-Intron6 junction. In this way, s-Nramp6 encodes a truncated OsNRAMP6 protein (amino acids 1 to 210) containing a C-terminal extension of seven amino acids (shown in the figure). Arrows denote the position of primers used for expression analysis in B. Gray bars represent the 5' and 3' untranslated regions (UTR). B, Accumulation of the l-Nramp6 and s-Nramp6 transcripts in leaves of rice (Oryza sativa Nipponbare) plants at different developmental stages. Reverse transcription-quantitative polymerase chain reaction was carried out using the OsUbiquitin1 (Os06g46770) gene as the internal control. Values represent the mean \pm standard error of three biological replicates. C, Alignment of 1-NRAMP6 and s-NRAMP6 amino acid sequences. Transmembrane domains (I to IX) were predicted using the SOSUI program (Hirokawa et al. 1998) and are indicated by thick solid lines above amino acid sequences. The 7 amino-acid extension at the C terminus of s-NRAMP6 (underlined) and the consensus transport sequence motif (CTM, dashed line) are shown. Dots indicate metal-coordinating residues within the metal-binding site (solid gray bar; $\mathrm{D}_{88} \mathrm{PSN}_{91}$ ) based on the homology to the bacterial NRAMP Staphylococcus capitis DMT (ScaDMT) transporter (these residues were reported to be involved in NRAMP metal binding and selectivity of ScaDMT) (Ehrnstorfer et al. 2014; Pottier et al. 2015). 
expressing $s$-NRAMP6-GFP, indicating an irregular distribution of s-NRAMP6 in the plasma membrane (Fig. 3F).

From these results, it is concluded that the two NRAMP6 protein isoforms (1-NRAMP6 and s-NRAMP6) localize at the plasma membrane. In addition, 1-NRAMP6 also accumulated in vesicles in the vicinity of the plasma membrane. Whether these vesicles represent an anterograde or retrograde trafficking of 1-NRAMP6 remains to be determined. Most importantly, this study reveals that s-NRAMP6 has the molecular determinants that are required to target OsNRAMP6 to the plasma membrane.

\section{OsNRAMP6 complements yeast mutants defective} in Fe and Mn transport.

NRAMP proteins from higher plants have been shown to rescue the phenotype of yeast mutants impaired in metal transport activities (Cailliatte et al. 2009; Curie et al. 2000; Ishimaru et al. 2012; Lanquar et al. 2005; Thomine et al. 2000; Xia et al. 2010; Yang et al. 2013; Yamaji et al. 2013). We used this approach to investigate the metal transport activity of 1-NRAMP6 and to investigate whether s-NRAMP6 is a functional metal transporter. For expression in yeast, each cDNA was cloned under the control of the constitutive promoter $A D H 1$ into the pWS93 vector, which allows the production of hemaglutinin (HA) epitope-tagged proteins. Western blot analysis of protein extracts from wildtype cells transformed with the HA-NRAMP constructs confirmed the accumulation of both 1-NRAMP6 and s-NRAMP6 in the insoluble protein fraction, as expected for a plasma membrane localization (Fig. 4A).

As a first step to investigate the metal transport activity of OsNRAMP6, the cDNA encoding either the long or the short NRAMP isoform was expressed in the fet 3 fet 4 yeast mutant, which is defective in both high- and low-affinity Fe uptake systems (Portnoy et al. 2000). Due to reduced Fe uptake, this mutant requires addition of significant amounts of $\mathrm{Fe}$ to the medium for growth (up to $150 \mu \mathrm{M}$ ) (Fig. 4B). We tested the ability of yeast transformants to grow on media supplemented with Fe in a wide range of concentrations ( 5 to $200 \mu \mathrm{M} \mathrm{Fe}$ ). Expression of l-OsNRAMP6 improved growth of the yeast fet 3 fet 4 mutant on medium containing amounts of $\mathrm{FeCl}_{3}$ as low as $5 \mu \mathrm{M}$ (Fig. 4B; for simplicity, only results obtained using 10 and $150 \mu \mathrm{M} \mathrm{FeCl}_{3}$ are presented), suggesting that 1-NRAMP6 protein is an Fe transporter. Interestingly, s-NRAMP6 also rescued growth of the fet 3 fet4 mutant at the same level (Fig. 4B), supporting the idea that the short NRAMP6 protein is a functional isoform.

Next, we investigated the ability of each NRAMP6 protein to transport Mn by functional complementation of yeast strains carrying deletions of the SMF1 and SMF2 genes. The SMF (suppressor of mitochondria import function) proteins are the yeast NRAMP homologs and act in Mn uptake. The growth of yeast smf mutant strains and the isogenic wild-type, transformed with pWS93 vector (negative control), l-NRAMP6, or s-NRAMP6, was analyzed in Mn-limited medium, which was controlled by addition of increasing concentrations of the divalent cation chelator ethylene glycol tetra-acetic acid. Results obtained indicated that both 1-NRAMP6 and s-NRAMP6 isoforms rescued, to some extent, the growth of smfl and smf2 yeast mutants under Mn-limited condition (Fig. 4C). Thus, yeast complementation studies indicated that the two protein isoforms 1-NRAMP6 and s-NRAMP6 are involved in Fe and Mn transport.

$\mathrm{Cd}$ and As transport activities have been described for several NRAMP proteins (Cailliatte et al. 2009; Ishimaru et al. 2012; Sasaki et al. 2012; Takahashi et al. 2011; Thomine et al. 2000; Tiwari et al. 2014). Here, we tested the sensitivity to $\mathrm{Cd}$ and As in wild-type yeast cells expressing either l-NRAMP6 or $s$-NRAMP6. No significant difference in toxicity was observed in synthetic medium supplemented with Cd or As (Supplementary Fig. S3), indicating that OsNRAMP6, most probably, does not mediate Cd or As transport in yeast.

Collectively, these results indicated that no significant differences in metal transport specificity or activity exist between
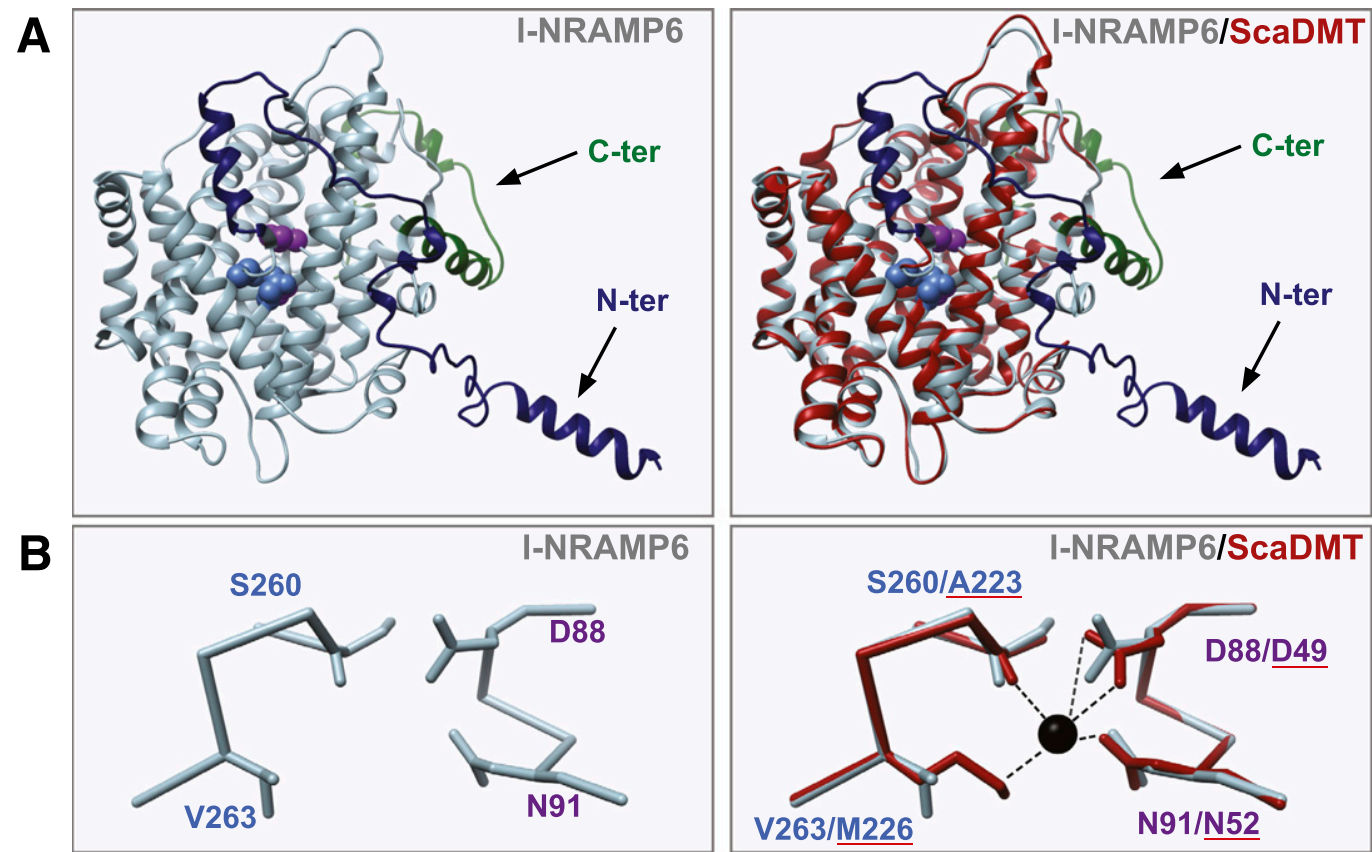

Fig. 2. Molecular modeling of OsNRAMP6. A, Predicted three-dimensional structure of NRAMP6 (1-NRAMP6) by homology-based modeling. The model was generated by I-TASSER (Roy et al. 2010) using the Staphylococcus capitis DMT (ScaDMT; Protein Data Back code 4WGW) structure as template (Ehrnstorfer et al. 2014). Molecular graphics images were produced using the UCSF Chimera package (Pettersen et al. 2004). The NRAMP6 structure is shown on the left and the structural superposition of NRAMP and ScaDMT is shown on the right. Residues involved in metal coordination are shown as spheres. B, Close-up view of the ion-binding site of 1-NRAMP6 (left). Residues involved in metal coordination were inferred from structural alignment of 1-NRAMP6 with ScaDMT. A close-up view alignment of the 1-NRAMP6 and ScaDMT ion-binding sites is shown (right). Residues D88 and N91 in NRAMP6 correspond to D49 and N52 in ScaDMT (underlined); S260 and V263 in NRAMP6 correspond to A223 and M226 in ScaDMT (underlined). Interactions are indicated by dashed lines and the metal ion is shown as a sphere. 


A pB7FWG2

B
I-NRAMP6

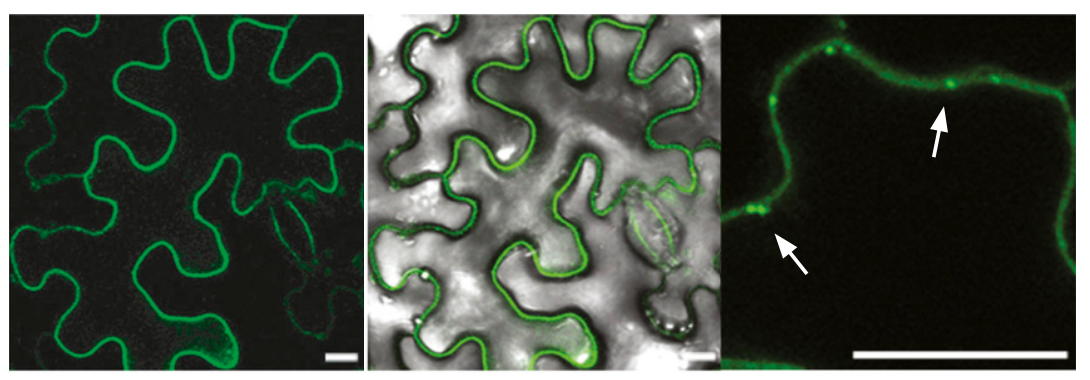

C
s-NRAMP6

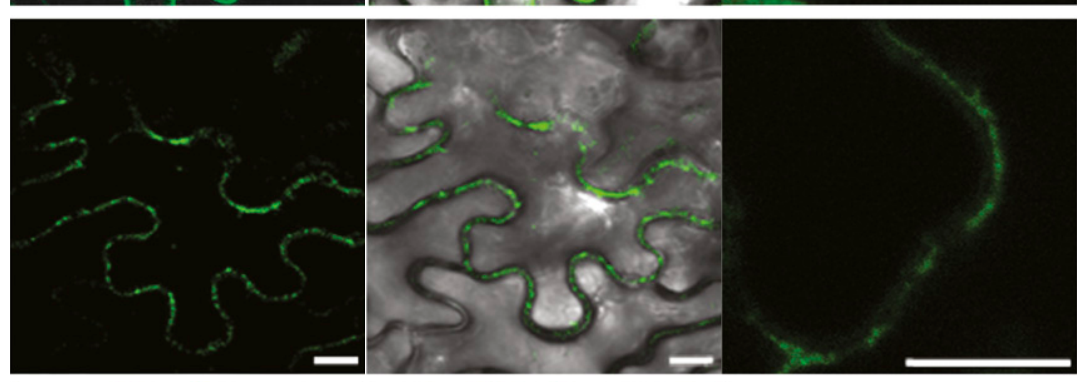

D

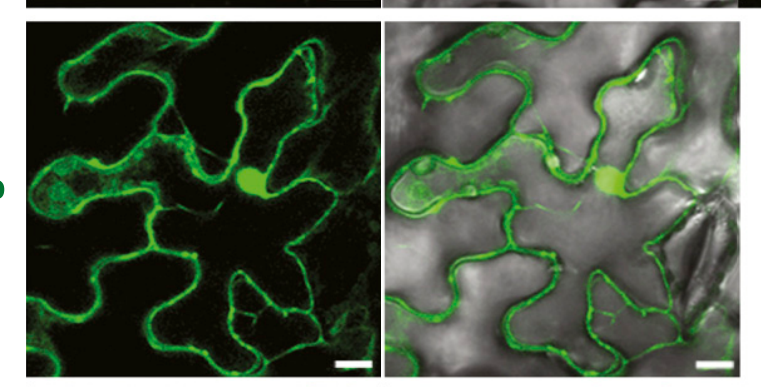

E
I-NRAMP6
+LTIB6

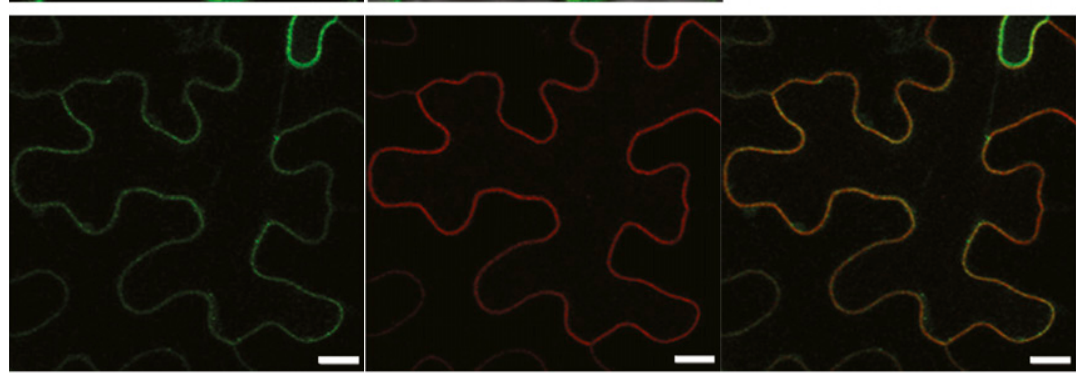

$\mathbf{F}$

S-NRAMP6 +LTIB6

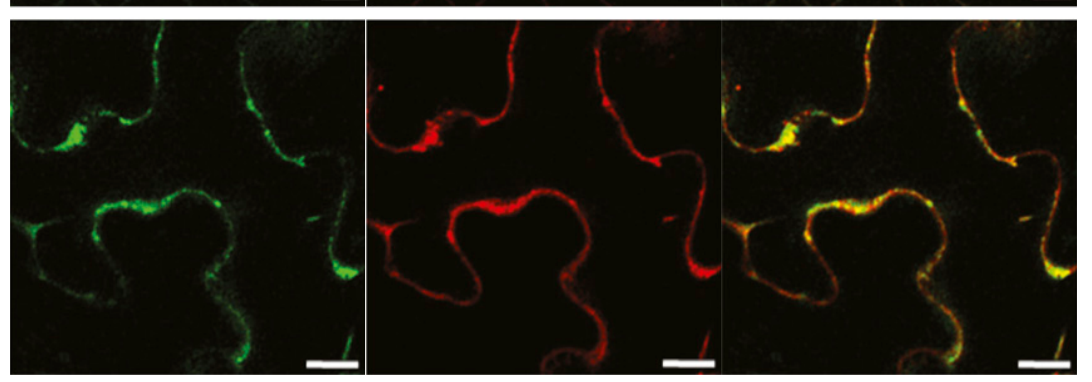

Fig. 3. Plasma membrane localization of OsNRAMP6. Confocal fluorescence microscopy of Nicotiana benthamiana leaves transiently expressing $l$-NRAMP6green fluorescent protein $(G F P)$ or s-NRAMP6-GFP fusion genes. A, Schematic representation of the plasmids used for transient expression of Nramp6-GFP fusion genes. OSNRAMP6 cDNA were fused to the N-terminal of GFP and expressed under the control of the constitutive Cauliflower mosaic virus $35 \mathrm{~S}$ promoter (35S). B and C, Cells expressing B, l-Nramp6-GFP or C, s-Nramp6-GFP. From left to right: GFP channel, overlay of GFP and bright-field channel, and magnification images. Arrows indicate discrete regions in the periphery and vesicles exhibiting strong GFP fluorescence. D, Cells expressing GFP. E and F, Cells coexpressing E, $l$-Nramp6-GFP or the F, $s$-Nramp6-GFP with the plasma membrane marker RFP-LTI6b. From left to right: GFP channel, red fluorescent protein (RFP) channel, and overlay of GFP and RFP channel. Confocal images were taken at $52 \mathrm{~h}$ after agroinfiltration. Individual sections are shown. Scale bars correspond to $10 \mu \mathrm{m}$. 
A

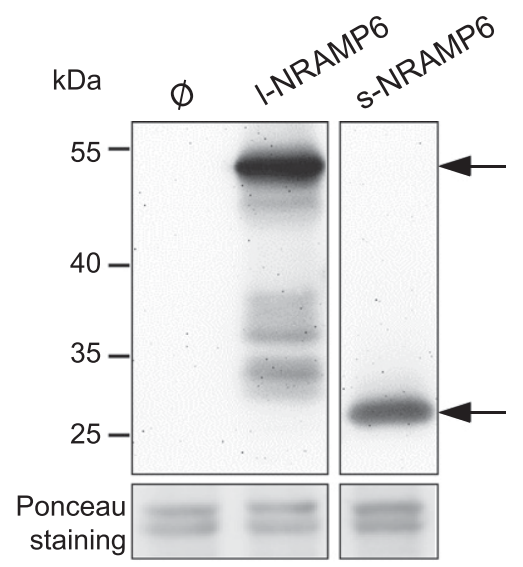

B

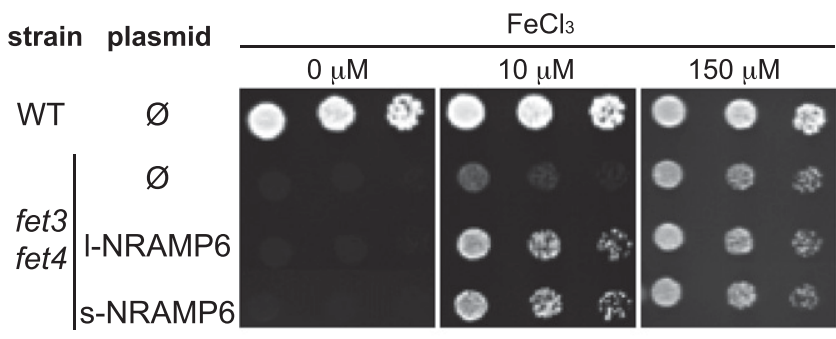

C

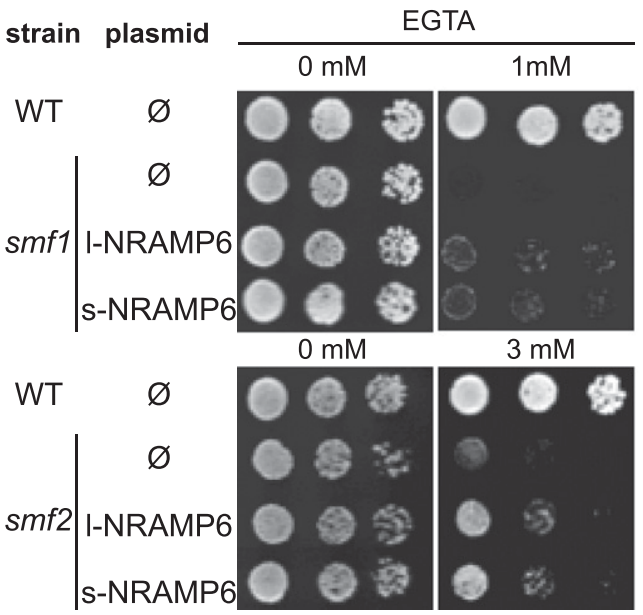

Fig. 4. NRAMP6 functions in Fe and Mn transport in yeast. A, Production of NRAMP6 proteins in yeast. The wild-type (WT) strain BY4741 was transformed with plasmid $p$ WS93/l-Nramp6 and $p$ WS93/s-Nramp6 which encoded hemaglutinin (HA)-tagged 1-NRAMP6 and s-NRAMP6 proteins, respectively, and grown on synthetic minimal medium (lacking uracil) until the exponential phase. Cultures were taken, and protein extracts were prepared and subjected to fractionation by centrifugation. Similar amounts of proteins from the insoluble fraction were subjected to $8 \%$ sodium dodecyl sulfate polyacrylamide gel electrophoresis followed by immunoblotting using the anti-HA antibody. A PageRuler prestained protein ladder (Thermo Fisher) was used. Arrows denote NRAMP6 proteins. B and C, Complementation assays in yeast mutant strains deficient in $\mathbf{B}$, the uptake of Fe (fet 3 fet 4 ) or $\mathbf{C}$, Mn transport (smfl and smf2). The WT strain BY4741 and the yeast mutants transformed with pWS93 empty vectors ( $)$ ) were used as positive and negative controls, respectively. B, Complementation of fet 3 fet 4 was evaluated by spotting transformed yeast cells at an optical density at $600 \mathrm{~nm}=0.05$, plus two 10 -fold dilutions, on synthetic minimal medium (lacking uracil) agar plates without or with $\mathrm{FeCl}_{3}$ supplementation. Growth was monitored after 3 days at $28^{\circ} \mathrm{C}$. C, Complementation of the smf1 and smf2 deletions was scored on low-YPD (yeast-peptone-dextrose) medium containing $50 \mathrm{mM}$ MES ( $\mathrm{pH} \mathrm{6)}$ ) agar plates supplemented with $1 \mathrm{mM}(s m f 1)$ or $3 \mathrm{mM}(s m f 2)$ ethylene glycol tetra-acetic acid (EGTA). Growth was monitored after 3 days at $28^{\circ} \mathrm{C}$.
1-NRAMP6 and s-NRAMP6 expressed in yeast. Of interest, this study also demonstrated that s-NRAMP6 is a functional protein that targets to the specific subcellular compartment in which 1-NRAMP6 also localizes.

\section{Phylogenetic relationships between members} of the rice and Arabidopsis NRAMP family.

Knowing that OsNRAMP6 functions as an Fe and Mn transporter, we searched for similarities or differences in metal transport activity of NRAMP proteins in the context of their phylogenetic relationship. For this, a phylogenetic tree was constructed using rice and Arabidopsis NRAMP protein sequences. Historically, the rice NRAMP family of metal transporters has been considered to contain seven members but the rice genome contains an additional Nramp gene, OsNramp8, that encodes a shorter, still-uncharacterized NRAMP protein. The Arabidopsis ETHYLENE INSENSITIVE2 protein shares sequence identity at its $\mathrm{N}$ terminus with NRAMP proteins (Alonso et al. 1999; Jun et al. 2004) and was included as an outroot.

The rice and Arabidopsis NRAMP proteins distributed into two phylogenetic groups (Fig. 5), which is consistent with results previously reported (de Carvalho Victoria et al. 2012; Gross et al. 2003). When examining the metal transport activity of Arabidopsis and rice NRAMP, we noticed that closely related NRAMP proteins might have different activities. For instance, both OsNRAMP1 and OsNRAMP5 function as Fe and Cd transporters, and clustered in group I. Of these, OsNRAMP5 (but not OsNRAMP1) is also involved in Mn transport, whereas OsNRAMP1 (but not OsNRAMP5) functions in Zn transport. Also, the closely related AtNRAMP1 and AtNRAMP6 proteins (group I) function in Cd transport but only AtNRAMP1 functions as an Fe and Mn transporter. Finally, AtNRAMP3 and AtNRAMP4 proteins (group II) are $\mathrm{Fe}, \mathrm{Mn}$, and $\mathrm{Cd}$ transporters but only AtNRAMP4 exhibits Zn transport activity. Altogether, these observations indicate that NRAMP within a particular phylogenetic clade might have different metal transport properties and points to an important specialization of NRAMP proteins in metal transport activity. This fact makes it difficult to predict transport activities of NRAMP based solely on protein sequence homology, and experimental evidence is needed to unravel the metal transport properties of individual NRAMP proteins.

\section{Regulation of Nramp6 expression during infection with the rice blast fungus $M$. oryzae.}

We previously reported that miR7695, a novel miRNA from rice, negatively regulates $s$-Nramp6 expression (Campo et al. 2013). Among the various transcript variants that are produced from the OsNramp6 gene by alternative splicing, only $s$-Nramp6 contains the binding site for miR7695, which is located at the $3^{\prime}$ untranslated region of the $s$-Nramp6 transcripts. Overexpression of miR7695 in rice was found to confer resistance to infection by the rice blast fungus $M$. oryzae. It was then of interest to investigate $O s N r a m p 6$ expression during $M$. oryzae infection.

Expression analysis revealed that fungal infection transiently increased accumulation of $s$-Nramp6 transcripts (at $24 \mathrm{~h}$ postinoculation [hpi]) and then returned to normal levels (48 and $72 \mathrm{hpi}$ ) (Fig. 6A, left panel) in leaves of rice (O. sativa 'Nipponbare') plants. As for l-Nramp6, its expression was slightly but significantly downregulated at 48 hpi with $M$. oryzae (Fig. 6A, right panel). To confirm that the plant detects and responds to pathogen infection, we examined pathogenesis-related-1a (PRla) expression, the PRla gene being considered a marker gene of defense gene activation during infection of rice plants with $M$. oryzae (Agrawal et al. 2001). As expected, fungal infection strongly induced PRla expression (Supplementary Fig. S4A).

Next, we investigated whether $M$. oryzae infection is accompanied by alterations in Fe or Mn content in rice leaves. Metal 
content of leaves of $M$. oryzae-infected and mock-inoculated plants was determined by inductively coupled plasma optical emission spectrometry (ICP-OES). This analysis revealed a small, transient increase in Fe content at 24 hpi with M. oryzae (Fig. 6B). Fungal infection, however, does not appear to have an important effect on $\mathrm{Mn}$ accumulation during the same period (Fig. 6B).

Collectively, this study revealed that $l$-Nramp6 and $s$-Nramp6 expression is regulated during infection of rice plants with the fungal pathogen $M$. oryzae. During the infection process, alterations in $s$-Nramp6 expression occur earlier than those in l-Nramp6. Fungal infection is accompanied by a slight and transient increase in Fe content in rice leaves whereas Mn content appears not to be affected by fungal infection, at least at the time points of infection examined here.

\section{Fe supply has an impact on resistance to infection by the rice blast fungus $M$. oryzae.}

Having established that OsNramp6 is regulated during infection with the rice blast fungus and that NRAMP6 functions as an Fe transporter, we investigated whether Fe supply has an effect on blast resistance. Toward this end, the rice plants were grown in hydroponic culture on half-strength Kimura B solution containing $10 \mu \mathrm{M}$ Fe-EDTA (control condition), low Fe $(0.1 \mu \mathrm{M}$ Fe-EDTA), or high Fe (1 mM Fe-EDTA) conditions. The effectiveness of Fe treatment was assessed by determining the expression of the Fe deficiency-responsive genes NASl, $I R O 2$, and IRT2 in rice roots. As expected, the three marker genes were upregulated and downregulated when grown under low-Fe and high-Fe conditions, respectively.
Plants at the four-leaf stage were inoculated with $M$. oryzae spores or mock inoculated and monitored for blast disease symptoms. Plants that had been grown under high Fe exhibited resistance to $M$. oryzae infection compared with plants grown under control conditions (Fig. 6C). In contrast to this, plants grown in low Fe showed higher susceptibility to blast than control plants. Scoring of symptom severity was performed using the 1-to-9 scale of the Standard Evaluation System for rice, where $1=$ no symptom and $9=$ highly susceptible. Symptom scale 4 was the typical response of plants grown under control Fe whereas symptom scales 3 and 6 were observed for rice plants under high or low Fe, respectively (Fig. 6C, upper panel). Determination of the leaf area covered by blast lesions and quantification of fungal DNA, an indicator of fungal biomass in the infected leaves, confirmed resistance to blast infection in high-Fe rice plants and susceptibility in low-Fe rice plants (Fig. 6C, lower panels).

We also examined the expression of defense-related genes in $M$. oryzae-inoculated and mock-inoculated plants grown under different Fe supply (at 24 and 48 hpi). As expected, PRla and $P R 10 b$ expression was induced in response to fungal infection (Fig. 6D, upper and middle panels). Consistent with the observed phenotype of resistance to $M$. oryzae infection, stronger induction of PRIa and PRIOb expression occurred in plants grown under high Fe compared with plants grown under control or low Fe (Fig. 6D). As for the rice plants grown under low $\mathrm{Fe}$ (increased susceptibility), we noticed that $P R 1 O b$ showed a weaker induction at 48 hpi compared with control and high-Fe plants (Fig. 6D, middle panel). However, susceptibility to blast infection in low-Fe plants was not accompanied by a lower level of PRI a expression compared with control plants, at least at
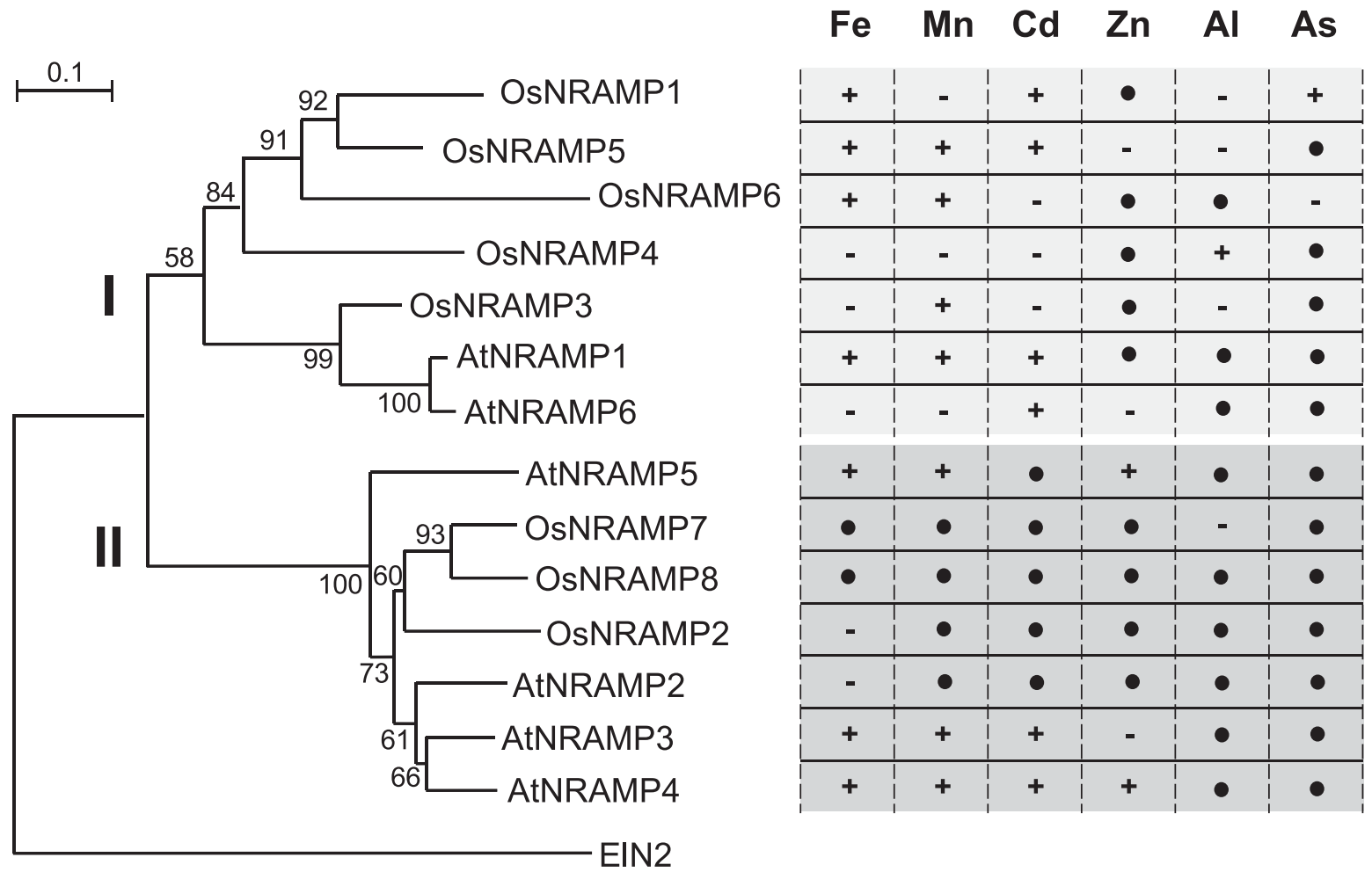

Fig. 5. Phylogenetic analysis and metal transport activity of rice and Arabidopsis NRAMP proteins. The phylogenetic tree of rice (Os) and Arabidopsis thaliana (At) NRAMP proteins was constructed with Molecular Evolutionary Genetics Analysis (MEGA 4.0) (Tamura et al. 2007) using Blossum matrix and the neighbor-joining method (Saitou and Nei 1987). Accession numbers for the corresponding genes are OsNramp1 (Os07g15460), OsNramp2 (Os03g11010), OsNramp3 (Os06g46310), OsNramp4 (Os02g03900), OsNramp5 (Os07g15370), OsNramp6 (Os01g31870), OsNramp7 (Os12g39180), OsNramp8 (Os03g41070), AtNramp1 (At1g80830), AtNramp2 (At1g47240), AtNramp3 (At2g23150), AtNramp4 (At5g67330), AtNramp5 (At4g18790), AtNramp6 (At1g15960), and AtEIN2 (At5g03280). The metal transport activity of the various NRAMP was previously described (Cailliatte et al. 2009; Curie et al. 2000; Ishimaru et al. 2012; Lanquar et al. 2004; Sasaki et al. 2012; Takahashi et al. 2011; Thomine et al. 2000; Tiwari et al. 2014; Xia et al. 2010; Yamaji et al. 2013; Yang et al. 2013). Symbols: $+=$ transport activity, $-=$ no transport activity, and $\mathbf{O}=$ not determined. 
the time points of infection analyzed here (Fig. 6D). A more detailed investigation of the PRla expression profile during infection is required to determine whether fungal responsiveness of this gene is repressed under low Fe supply.

It is well known that, during pathogen infection, there is an increase in ROS production, including hydrogen peroxide $\left(\mathrm{H}_{2} \mathrm{O}_{2}\right)$. However, when in excess, ROS cause a state of oxidative stress in the plant capable of injuring cells by oxidation of biomolecules (e.g., DNA, proteins, and lipids) and, ultimately, cell death. Apoplastic peroxidases, which convert $\mathrm{H}_{2} \mathrm{O}_{2}$ to water, play a central role in protecting the plant cell from oxidative damage, because $\mathrm{H}_{2} \mathrm{O}_{2}$ is a relatively long-lived molecule highly permeable across membranes. Taking into consideration results previously reported, indicating that Fe accumulates in the apoplast during pathogen attack and that the $\mathrm{Fe}^{2+} / \mathrm{Fe}^{3+}$ redox cycling generates ROS (Liu et al. 2007), we sought to investigate the expression of the apoplastic peroxidase POC1 (Os07g48050) gene in rice plants grown under different Fe supply. $P O C 1$ is also known to be induced in plants in response to pathogen infection, including rice plants (Anguelova-Merhar et al. 2002; Young et al. 1995). POC1 expression was induced to a higher level in leaves of rice plants that have been grown in high $\mathrm{Fe}$ and inoculated with
A

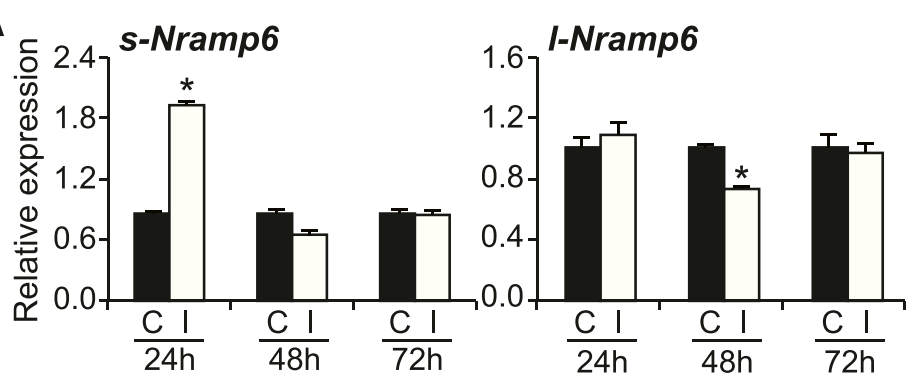

C
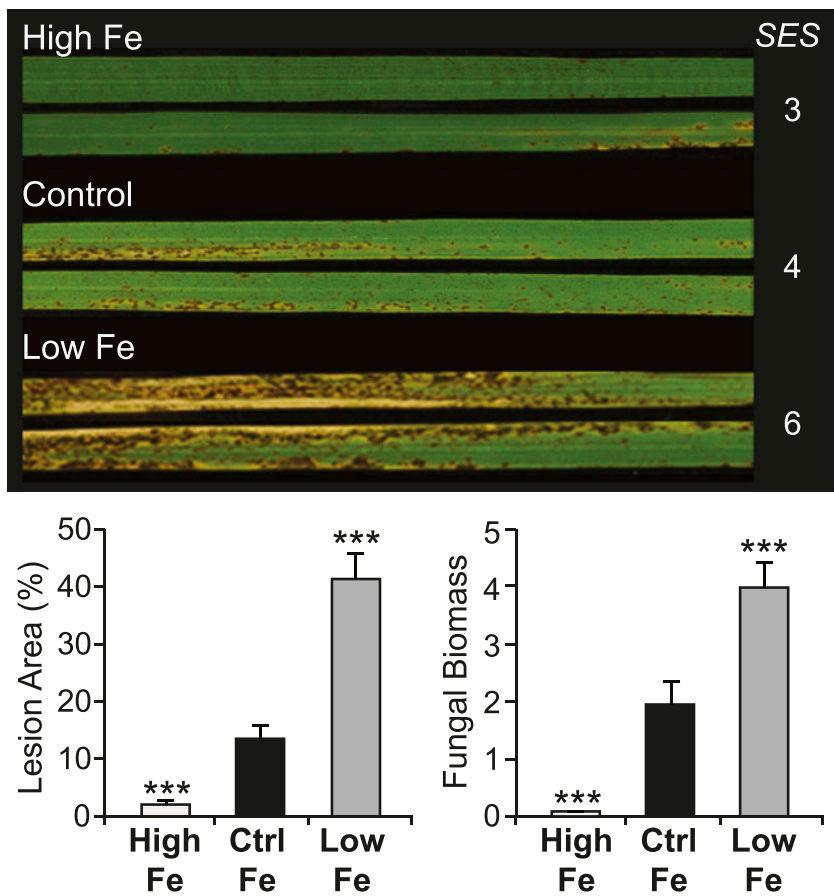

B

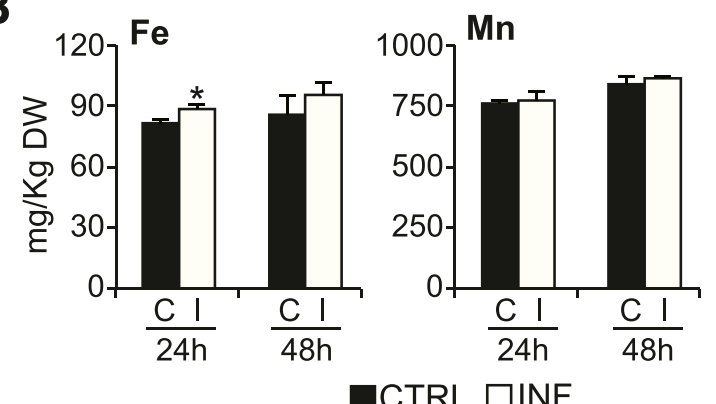

D

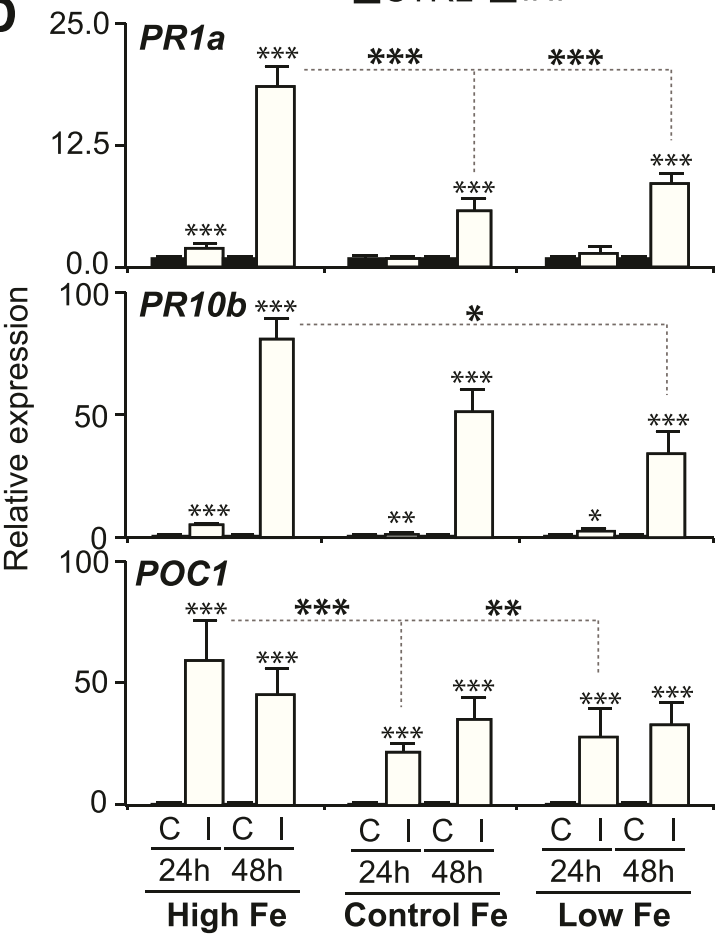

Fig. 6. Alterations in Fe content in rice plants during infection with Magnaporthe oryzae and influence of Fe supply on disease resistance. A, Accumulation of l-Nramp6 and s-Nramp6 transcripts in leaves of M. oryzae-inoculated (I) and mock-inoculated (C) rice (Oryza sativa Nipponbare) plants at the indicated times after inoculation. Reverse transcription-quantitative polymerase chain reaction (RT-qPCR) was carried out using the OsUbiquitin1 gene (Os06g46770) as the internal control. Values represent means normalized to its corresponding control for each time point, arbitrarily set to $1 \pm$ standard error $(\mathrm{SE})(n=3$, each biological replicate is a pool of 5 individual leaves). B, Fe and Mn content in M. oryzae-infected rice leaves. Fe (left panel) and Mn (right panel) content was determined by inductively coupled plasma optical emission spectrometry in leaves of $M$. oryzae-inoculated and mock-inoculated rice plants at the indicated times after inoculation. Values represent the mean $\pm \mathrm{SE}$ ( $n=3$, each biological replicate is a pool of 5 individual leaves). $\mathbf{C}$, Effect of Fe supply on resistance to $M$. oryzae infection. Plants were grown for 15 days in hydroponic cultures (half-strength Kimura B solution), treated during 4 days more with the same solution (control = $10 \mu \mathrm{M}$ Fe-EDTA), low Fe $(0.1 \mu \mathrm{M} \mathrm{Fe})$, or high Fe $(1 \mathrm{mM} \mathrm{Fe})$ and then sprayed with a spore suspension of $M$. oryzae $\left(5 \times 10^{5}\right.$ spores/ml $)$. Disease symptoms at 7 days postinoculation are shown. Numbers on the right indicate the response to $M$. oryzae infection according to the Standard Evaluation System $(\mathrm{SES})$ for rice $(0=$ no visible lesions and $9=$ highly susceptible). Percentage of leaf area affected by blast lesions was determined by image analysis (APS Assess 2.0) (left lower panel). Relative quantification of $M$. oryzae DNA was determined by qPCR using specific primers of the $M$. oryzae 28S ribosomal gene relative to the rice Ubiquitin1 gene (Os06g46770) (right lower panel). Values represent the mean $\pm \mathrm{SE}(n=8)$. Results are from one of two independent experiments which gave similar results. D, Expression of defenserelated genes in rice plants that have been grown under control, low, or high Fe supply. qRT-PCR was used to monitor the expression of the defense marker genes PRla (Os07g03710), PR1Ob (Os12g36850), and POC1 (Os07g48050), encoding the apoplastic peroxidase POC1, using the OsUbiquitin1 gene (Os06g46770) as the internal control. Values represent means normalized to its corresponding control for each time point, arbitrarily set to $1 \pm \operatorname{SE}(n=3$, each biological replicate is a pool of 5 individual leaves). Asterisks indicate significant differences between treatments (analysis of variance: $*, * *$, and $* * *$ indicate $P<0.05,0.01$, and 0.001 , respectively). 
M. oryzae ( $24 \mathrm{hpi})$ compared with the fungal-inoculated plants grown in either low or control conditions (Fig. 6D).

Collectively, these studies revealed that infection of rice plants grown under high Fe supply results in stronger induction of defense-related genes, notably PRla, PRIOb and POCl, which is in agreement with the observed phenotype of resistance to $M$. oryzae infection in these plants. However, rice plants grown in low $\mathrm{Fe}$ show enhanced susceptibility to $M$. oryzae, but expression of the defense gene here examined appears not to be significantly affected (only $P R I O b$ is less induced in low Fe than in control conditions, at 48hpi).

\section{Osnramp6 mutant plants exhibit resistance to infection by $M$. oryzae.}

The importance of OsNramp6 in disease resistance was investigated by characterizing the response to $M$. oryzae infection in nramp6 mutant plants. A transfer-DNA (T-DNA) insertion line produced in the 'Hwayoung' genotype was identified in the POSTECH Rice Insertion Database (RISD) (2B-20317). As was previously found in Nipponbare rice, OsNramp6 was expressed in leaves of Hwayoung plants, the l-Nramp6 transcripts accumulating at higher level than the s-Nramp6 transcripts (Supplementary Fig. S5). The 2B-20317 rice mutant contains a T-DNA inserted in intron 12 of OsNramp6 (Fig. 7A). PCR analysis using combinations of gene-specific and T-DNA-specific primers followed by sequencing of PCR products confirmed the T-DNA insertional locus in the 2B-20317 mutant (Fig. 7A). qPCR revealed that this mutant had a single copy of T-DNA inserted in its genome (results not shown). Moreover, l-Nramp6 transcripts were found to be absent in the nramp6 mutant, thus supporting the idea that this is a knock-out mutant for l-Nramp6 (Fig. 7B). However, accumulation of $s$-Nramp6 transcripts remained unaltered in these plants, pointing to a cotranscriptional production of s-Nramp6 transcripts during OsNramp6 transcription in nramp6 plants (Fig. 7B). In this context, though limited information is currently available regarding cotranscriptional splicing in plant cells, studies in other organisms (e.g., yeast, Drosophila, human, and mouse cells) demonstrated that the majority of splicing events take place cotranscriptionally (Brugiolo et al. 2013).

To determine the role of OsNramp6 in plant defense, wildtype azygous and nramp6 mutant plants were infected with M. oryzae. Results obtained indicated that nramp6 plants displayed enhanced resistance to $M$. oryzae infection compared with control azygous plants, as determined by visual inspection and quantification of the leaf area covered by blast lesions (Fig. 7C). These findings support the idea that OsNramp6 negatively regulates resistance to the rice blast fungus in rice plants.

Finally, we investigated whether OsNramp6 has a role in growth and development in rice. For this, wild-type and nramp6 plants were grown in hydroponic culture in control conditions for 6 weeks. Root, stem, and leaf biomass was determined. Results obtained show that nramp6 mutant plants had reduced biomass compared with wild-type plants (azygous, segregated from heterozygous plants) (Fig. 8A). Biomass reduction was more evident in root tissues of nramp6 plants (Fig. 8A), also supported by a reduction in root length (Fig. 8B). Although no significant reduction in biomass was observed in leaves, a reduction in length of the youngest leaves was observed for nramp6 in mutant plants. Altogether, these observations point to a role of OsNramp6 in plant growth. However, further studies are needed to determine the exact role of OsNramp6 in controlling rice growth and development.

\section{DISCUSSION}

We report the functional characterization of OsNramp6, a member of the rice NRAMP gene family that mediates pathogen resistance. Both the 1-NRAMP6 and s-NRAMP6 protein produced by alternative splicing of primary Nramp6 transcripts function as $\mathrm{Fe}$ and $\mathrm{Mn}$ transporters, as revealed by functional complementation of yeast mutants defective in metal transport.

\section{A \\ OsNramp6 (Os01g31870.1)}
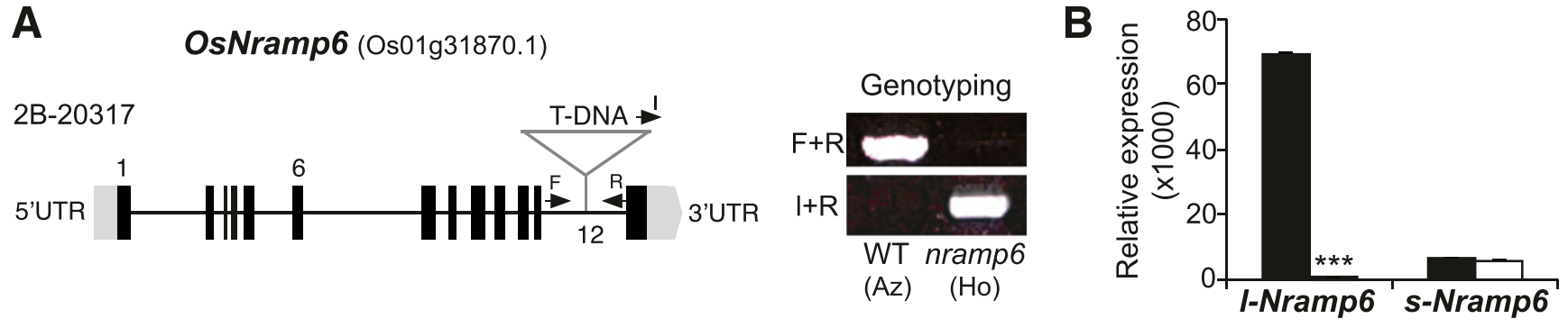

C
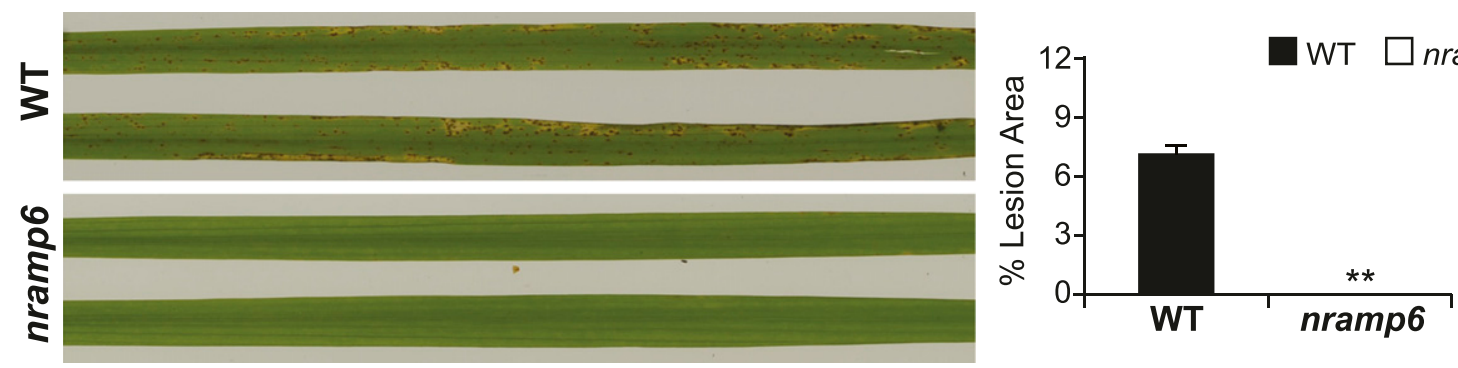

Fig. 7. OsNramp6 silencing confers resistance to infection by the rice blast fungus Magnaporthe oryzae. A, Schematic representation of the T-DNA insertion in OsNramp6 (2B-20317, POSTECH collection). Arrowheads indicate the position of primers used for polymerase chain reaction (PCR) genotyping. Verification was done by sequencing of the amplification fragments. WT $=$ segregated azygous (Az) plant and Ho = homozygous. B, Accumulation of $l$-Nramp6 and s-Nramp6 transcripts in nramp6 mutant plants (Hwayoung background) was performed by Reverse transcription-quantitative PCR, using the OsUbiquitin1 gene (Os06g46770) as the internal control. Values represent the mean \pm standard error (SE) (t-Student, *** indicates $P \leq 0.001)$. Data represent results obtained in two independent experiments that gave similar results. C, Resistance of nramp6 mutant plants to $M$. oryzae infection. Leaves of soil-grown plants at the fourleaf stage were sprayed with a $10^{5}$ spores/ml suspension $(0.2 \mathrm{ml} / \mathrm{plant})$. Disease symptoms at 7 days postinoculation are shown (left panel). Results are from one of three independent experiments (at least six plants/genotype), which gave similar results. Percentage of leaf area affected by blast lesions was determined by image analysis (APS Assess 2.0 PROGRAM) (right panel). Histograms show the mean $\pm \mathrm{SE}(n=6)$. Asterisks denote statistical differences between WT and nramp6 plants (t-Student; $* *$ and $* * *$ indicate $P \leq 0.01$ and 0.01 , respectively). 
We also show that both 1-NRAMP6 and s-NRAMP6 localize at the plasma membrane. In this respect, plasma membranetargeted NRAMP proteins are generally associated with metal uptake, whereas those located in intracellular membranes contribute to metal mobilization (Ishimaru et al. 2012; Lanquar et al. 2005; Sasaki et al. 2012; Thomine et al. 2003). For instance, the plasma membrane-localized OsNRAMP3 functions as a switch for regulating Mn distribution in the rice tissues for dealing with variable changes of $\mathrm{Mn}$ in the environment (Yamaji et al. 2013; Yang et al. 2013). Homology-based modeling revealed that the overall structure of NRAMP6 and ScaDMT is conserved, except for the $\mathrm{N}$-terminal and $\mathrm{C}$-terminal region. Whereas the binding-site aspartate and asparagine residues are shared by NRAMP6 and ScaDMT (D88/D49 and N91/N52 in 1-NRAMP6 and ScaDMT, respectively) (Fig. 2), the alanine and methionine residues in the metal binding site of ScaDMT (A223 and M226) are replaced by serine and valine in NRAMP6 (S260 and V263). An intriguing question arises from the observation that s-NRAMP6, which lacks S260 and V263, is functional in transporting $\mathrm{Fe}^{2+}$ and $\mathrm{Mn}^{2+}$. Here, it is worthwhile to recall that the ScaDMT structure consists of two symmetrically oriented subdomains (inverted repeats of five helices: helices 1 to 5 and 6 to 10), and that the two related halves gives rise to the ion channel in the center of the membrane (Ehrnstorfer et al. 2014). There is then the possibility that s-NRAMP6 forms active dimers or multimers. This hypothesis is supported by the observation of fluorescent aggregates in the plasma membrane of $s$-Nramp6-GFP expressing $N$. benthamiana cells. Further studies are required to determine whether NRAMP6 transports other metals not assayed

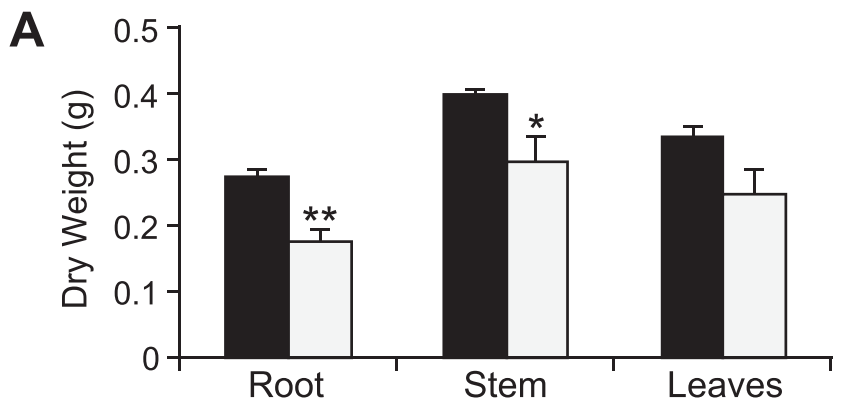

B

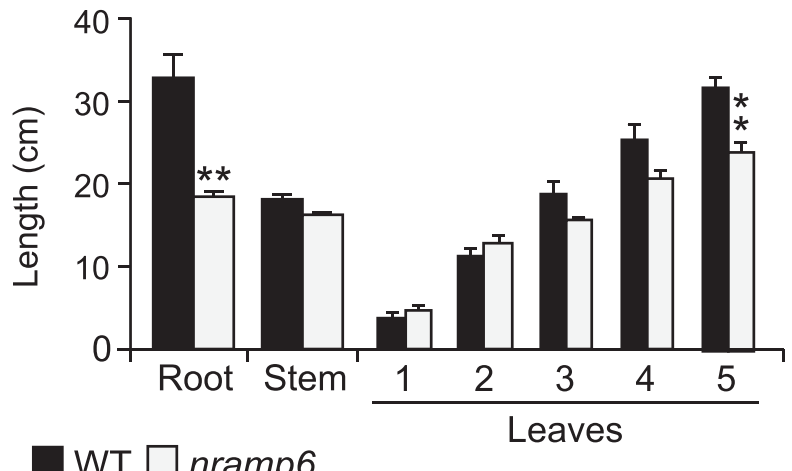

Fig. 8. Growth and biomass of nramp6 mutant plants. Wild-type (WT, segregated azygous) and nramp6 mutant plants were grown for 42 days in hydroponic cultures (half-strength Kimura B solution). Roots, stems, and leaves were collected separately. A, Dry weight of roots, stems, and leaves. Data represent the means \pm standard error (SE) $(n=4$, each biological replicate is a pool of 5 individual plants). B, Length of roots, stems, and leaves. Leaf length was measured for independent leaves, from number 1 (oldest) to number 5 (youngest). Data represent the means $\pm \operatorname{SE}(n=4)$ Two independent experiments were carried out with similar results. Asterisks denote statistically significant differences between WT and nramp6 plants (analysis of variance: $*$ and $* *$ indicate $P<0.05$ and 0.01 , respectively). in this work and to clarify the structural or mechanistic features underlying the observed metal transport activity of s-NRAMP6.

We show that $l$-Nramp6 and $s$-Nramp6 transcripts coexist in leaves of rice plants during vegetative growth and that their accumulation is regulated during infection with the foliar pathogen $M$. oryzae, although with different trend and kinetics. We also show that knockout nramp6 plants exhibited enhanced resistance to $M$. oryzae infection, indicating that OsNramp6 negatively regulates resistance to the rice blast fungus. Here, it is worthwhile to recall that, whereas l-Nramp6 expression is abolished in the nramp6 mutant, $s$-Nramp6 expression is not affected (Fig. 7B). In earlier studies, we reported that downregulation of $s$-Nramp6 transcripts through overexpression of miR7695 also confers resistance to infection by the rice blast fungus (Campo et al. 2013). Taken together, these results indicate that l-Nramp6 knock-out rice plants (present work) and rice plants in which s-Nramp6 is downregulated (miR7695 overexpressor rice plants) (Campo et al. 2013) exhibit enhanced resistance to pathogen infection. Disturbance on the accumulation of the two splicing transcripts l-Nramp6 or s-Nramp6 might trigger still unknown signaling processes mediating defense responses in rice plants. Recently, Liu and coworkers (2016) provided evidence for alternative splicing of rice WRKY transcription factors (TF) involved in resistance to the rice blast fungus $M$. oryzae. These authors also described a dominantnegative function of the truncated splice variants of these WRKY TF on the function of full-length OsWRKY proteins (Liu et al. 2016). Whether an alternative splicing-mediated feedback regulation mechanism occurs for the OsNRAMP6 proteins remains to be determined.

In the context of plant-pathogen interactions, metal homeostasis must be tightly controlled as host and pathogen compete for the available metals. The host plant must arrest pathogen invasion with minimal interference with normal growth and development. Plants might counteract pathogen infection by sequestering essential micronutrients away from the invading pathogen or by creating a controlled, localized accumulation of metals acting as antimicrobial agents. Indeed, the term "nutritional immunity", a process by which a host organism sequester minerals in an effort to limit pathogen infection, is used in humans and other mammals as a nonspecific host immune response against invading pathogens (Hood and Skaar 2012). In this respect, macrophage NRAMP proteins were originally described for their roles in regulating susceptibility to infectious and autoimmune disease in animals (Blackwell et al. 2003). On the other hand, $\mathrm{Fe}$ availability is also crucial for vegetative growth and virulence of $M$. oryzae (Hof et al. 2007, 2009). This fungus excretes siderophores of the coprogen type for $\mathrm{Fe}$ acquisition and uses ferricrocin as intracellular siderophore for Fe storage. In addition to acting as Fe chelators, siderophores provide protection against free radicals formed by $\mathrm{Fe}$, and M. oryzae mutants affected in siderophore biosynthesis exhibit increased sensitivity to oxidative stress (Hof et al. 2009). As previously mentioned, one of the earliest reactions to pathogen infection in plants is a burst in the production of ROS. Along with this, microbial siderophores might well function as modulators of plant defense responses (Dellagi et al. 2009).

Our data show that Fe supply has dramatic effects on resistance to $M$. oryzae infection in rice plants. Thus, growing rice plants in high $\mathrm{Fe}$ results in increased resistance to infection by the rice blast fungus $M$. oryzae. This resistance phenotype might be explained, at least in part, by the superinduction of defense gene expression (e.g., PRI $a$ and $P R 1 O b$ ). Conversely, rice plants grown in low $\mathrm{Fe}$ become more susceptible to $M$. oryzae infection. In other studies, however, Fe deficiency was found to increase resistance to the bacterial pathogen $D$. dadantii or the fungal pathogen Botrytis cinerea in Arabidopsis, both necrotrophic 
pathogens (Kieu et al. 2012). Thus, different infections phenotypes can be observed in Fe-starved plants. Whether this is due to different pathogen lifestyles remains to be determined.

Being a foliar pathogen, $M$. oryzae entirely depends on the host for supply of mineral nutrients. This fungus has a hemibiotrophic lifestyle that maintains an initial biotrophic relationship with its host, followed by a necrotrophic lifestyle (Campos-Soriano et al. 2012; Kankanala et al. 2007; Wilson and Talbot 2009). The availability or accessibility of host nutrients during $M$. oryzae infection has been shown to be an important factor contributing to the establishment of the rice blast disease (Wilson et al. 2012). In this work, we observed alterations in $s$-Nramp6 and l-Nramp6 expression during the M. oryzae biotrophic colonization phase (24 to $48 \mathrm{hpi}$ ). During this period, the fungus has no or limited access to intracellular metals, which are often sequestered in the vacuole for the plant's own use. Infection by $M$. oryzae is also accompanied by a transient and small increase in Fe content in rice leaves (at $24 \mathrm{hpi}$ ) whereas the Mn level appears not to be affected during fungal infection (at least at the time points assayed here). Here, it is worthwhile to recall that, although Fe is an essential element for plant growth, when present in excess, it is toxic. Under this scenario, a signaling function of NRAMP6-mediated alterations in $\mathrm{Fe}$ content in the infected tissue regulating rice immunity should be considered.

$\mathrm{Fe}$ is a potent generator of ROS, and signaling via ROS is widely regarded to be central to disease resistance in plants (Torres et al. 2006). A relationship of the Fe homeostasis with the production of ROS and disease resistance is documented in the literature (Expert et al. 2012; Fones and Preston 2012). The prevalent hypothesis is that localized and targeted redistribution of $\mathrm{Fe}$ in infected plant tissues mediates $\mathrm{H}_{2} \mathrm{O}_{2}$ production in the apoplast and contributes to immunity through activation of extracellular and intracellular defense responses. In particular, infection of wheat plants with Blumeria graminis f. sp. tritici has been shown to elicit the accumulation of $\mathrm{Fe}^{+3}$ in cell wall appositions at the infection site (Liu et al. 2007). The accumulated apoplastic $\mathrm{Fe}$ mediates oxidative burst with the production of apoplastic $\mathrm{H}_{2} \mathrm{O}_{2}$, thus inducing the expression of defense-related genes. Activity of the apoplastic peroxidase POC1 might well contribute to maintaining the $\mathrm{H}_{2} \mathrm{O}_{2}$ concentration in the apoplast at an appropriate level during $M$. oryzae infection. This would be consistent with the observation that, during $M$. oryzae infection, $P O C l$ is activated at a higher level in rice plants that have been grown under high $\mathrm{Fe}$ compared with control conditions. Transgenic expression of the rice peroxidase POCl in carrot was shown to confer resistance to fungal pathogens (Wally et al. 2009). Also, the expression of $P O C 1$ was found to be upregulated in transgenic rice plants containing the $P i 54$ gene exhibiting resistance to the rice blast fungus (Gupta et al. 2012), further supporting the important role of POC1 in plant immunity. The involvement of several peroxidases in apoplastic immunity is well documented in different plant-pathogen interactions (Doehlemann and Hemetsberger 2013), and peroxidases have been identified in the apoplastic proteome in infected plant tissues (Delaunois et al. 2014). Indeed, a proteomic analysis of the apoplastic secretome of the rice- $M$. oryzae interaction revealed that up to $10.25 \%$ of the apoplastic proteins comprise antioxidative or detoxifying proteins (Kim et al. 2013). On the other hand, because Fe serves as a cofactor for enzymes involved in ROS scavenging, Fe limitation might negatively impact ROS scavenging mechanisms during pathogen defense, which would then explain the observed phenotype of susceptibility in Fe-starved rice plants. The possibility that Fe deprivation influences metabolic processes or physiological functions that might negatively affect disease resistance in rice plants should be also considered. Now, it will be of interest to determine whether alterations in OsNramp6 expression are accompanied by a localized and targeted accumulation of $\mathrm{Fe}$ at the sites of infection for the control of host defense responses.

Different mechanisms, including NRAMP6 activity among other metal transporters, must operate in the plant to control $\mathrm{Fe}$ homeostasis as part of the plant response to pathogen infection. Given the high number of metal transporter families present in rice, it is plausible to consider that compensatory mechanisms might operate to deploy an effective defense response to pathogen infection. Candidates for compensation by other transporters are the closely related OsNRAMP1 and OsNRAMP5 (also Fe and Mn transporters), as well as OsIRT1 and OsYSL (implicated in Fe transport) (Inoue et al. 2009; Ishimaru et al. 2006, 2010). In favor of this hypothesis, previous studies have shown that OsNRAMP5, OsNRAMP1, OsIRT1, and OsYSL15 function jointly in Fe uptake in rice (Ishimaru et al. 2012).

Finally, we show that nramp6 mutant plants had less biomass than wild-type plants. Thus, in addition to disease resistance, OsNramp6 might also play a role during plant growth, as has been demonstrated for other Nramp genes (Cailliatte et al. 2010; Ishimaru et al. 2012; Lanquar et al. 2005; Sasaki et al. 2012; Yamaji et al. 2013). Growth-defense tradeoffs are known to occur in plants due to resource restrictions, which demand prioritization toward either growth or defense (Huot et al. 2014). Future studies are needed to establish possible interactions between Nramp6-mediated regulatory mechanisms controlling plant growth and defense responses.

In summary, results presented here demonstrated that Nramp6 is a negative regulator of disease resistance to the rice blast fungus, most probably through its activity as Fe (or Mn) transporter. In this respect, the intricate crosstalk between metal homeostasis and innate immunity in plants is just beginning to be understood. Because M. oryzae is one of the primary causes of rice losses worldwide, a better understanding of the mechanisms involved in $\mathrm{Fe}$ and $\mathrm{Mn}$ homeostasis in which Nramp6 participates will help in designing novel strategies to improve disease resistance in rice.

\section{MATERIALS AND METHODS}

\section{Plant material and growth conditions.}

Rice ( $O$. sativa subsp. japonica) plants were grown in soil at $28^{\circ} \mathrm{C}$ under a photoperiod cycle of $14 \mathrm{~h}$ of light and $10 \mathrm{~h}$ of darkness. For hydroponic cultivation, the rice seed were grown in half-strength Kimura B solution, as previously described (Sasaki et al. 2012). The composition of the nutrient solution was $0.18 \mathrm{mM}\left(\mathrm{NH}_{4}\right)_{2} \mathrm{SO}_{4}, 0.27 \mathrm{mM} \mathrm{MgSO} \cdot 7 \mathrm{H}_{2} \mathrm{O}, 0.09 \mathrm{mM}$ $\mathrm{KNO}_{3}, 0.18 \mathrm{mM} \mathrm{Ca}\left(\mathrm{NO}_{3}\right)_{2} \cdot 4 \mathrm{H}_{2} \mathrm{O}, 0.09 \mathrm{mM} \mathrm{KH}_{2} \mathrm{PO}_{4}, 8 \mu \mathrm{M}$ $\mathrm{MnCl}_{2} \cdot 4 \mathrm{H}_{2} \mathrm{O}, 3 \mu \mathrm{M} \mathrm{H} \mathrm{HO}_{3}, 1 \mu \mathrm{M}\left(\mathrm{NH}_{4}\right)_{6} \mathrm{Mo}_{7} \mathrm{O}_{24} \cdot 4 \mathrm{H}_{2} \mathrm{O}$, $0.4 \mu \mathrm{M} \mathrm{ZnSO} \cdot \cdot 7 \mathrm{H}_{2} \mathrm{O}, 0.2 \mu \mathrm{M} \mathrm{CuSO}_{4} \cdot 5 \mathrm{H}_{2} \mathrm{O}$, and $10 \mu \mathrm{M} \mathrm{Fe}-$ EDTA (pH 5.6) (Sasaki et al. 2012). To assess the effect of $\mathrm{Fe}$ supply, the hydroponically grown plants were transferred to the same nutrient condition containing either a lower Fe concentration $(0.1 \mu \mathrm{M}$ Fe-EDTA) or a higher Fe concentration $(1 \mathrm{mM}$ Fe-EDTA). Control plants continued to receive control nutrient solution. The nutrient solution was renewed every 3 days. After 4 days of treatment, plants were inoculated with a suspension of M. oryzae spores $\left(5 \times 10^{5}\right.$ spores $\left./ \mathrm{ml}\right)$. Disease symptoms were evaluated at 7 days postinoculation (dpi). Control and treated plants ( $n=8$ for each condition) were examined for biomass production by harvesting roots, stems, and leaves. Tissues were dried at $70^{\circ} \mathrm{C}$ for 3 days. For gene expression analysis, leaves were sampled and immediately frozen $\left(-80^{\circ} \mathrm{C}\right)$ at the time points analyzed.

The T-DNA insertion line for OsNramp6 (Os01g31870) and its wild-type genotype (O. sativa Hwayoung, japonica type) 
were obtained from the POSTECH RISD and grown as described above.

\section{Phylogenetic analysis.}

The cDNA and genomic sequences were retrieved from the Rice Genome Annotation Project database. Alignment of NRAMP amino acid sequences was carried out with ClustalW using default settings and Boxshade. The phylogenetic tree was constructed using the neighbor-joining algorithm by MEGA4 software (Tamura et al. 2007). TM domains were predicted with SOSUI (Hirokawa et al. 1998).

\section{Structure modeling.}

The three-dimensional structure of the long NRAMP6 was performed using, as a template, the ScaDMT protein (RCSB Protein Data Bank, code 4WGV) (Ehrnstorfer et al. 2014). The threedimensional model was built directly using the I-Tasser (version 3) on-line server (Roy et al. 2010) and then evaluated using the ProSA program (Sippl 1993; Wiederstein and Sippl 2007). The resulting protein structures were visualized using the UCSF Chimera package (Pettersen et al. 2004).

\section{RNA extraction and RT-qPCR.}

Total RNA was extracted from plant tissues using the Trizol reagent (Invitrogen). The first complementary DNA was synthesized from DNase-treated total RNA $(1 \mu \mathrm{g})$ (High Capacity cDNA Reverse Transcription kit; Life Technology). RT-qPCR was performed on optical 96-well plates (Light Cycler480; Roche Diagnostics) using SYBR Green dye and the primers listed in Supplementary Table S1. Primers were designed using Primer Express Software (Applied Biosystems). Data were normalized using the OsUbiquitin1 (Os06g46770) gene. Three independent biological replicates and three technical replicates per sample were analyzed.

\section{Transient expression of OsNramp6 in $N$. benthamiana leaves.}

The cDNA sequence encoding the full-length (Os01g31870.1) or the short (Os01g31870.8) NRAMP6 proteins were obtained from the Rice Genome Resource Center (J013135E06 and J013164K10 clones, respectively). The OsNramp6 cDNA sequence corresponding to l-Nramp6 or s-Nramp6 was amplified using the gw_l-Nramp6 and gw_s-Nramp6 primers (for l-Nramp6 and $s$-Nramp6, respectively). Each $0 s N$ ramp6 cDNA sequence was cloned into the $\mathrm{pB} 7 \mathrm{FWG} 2,0$ plant expression vector designed for the production of C-terminal GFP-tagged fusion proteins under the control of the $35 \mathrm{~S}$ Cauliflower mosaic virus promoter (Karimi et al. 2002). The plasmid construct containing either the l-Nramp6GFP or the $s$-Nramp6-GFP fusion gene was introduced into the Agrobacterium tumefaciens EHA105 strain. Agrobacterium-mediated transient expression assays were carried out in N. benthamiana leaves (rdr6i mutant) (Schwach et al. 2005). Experiments for coexpression with the fluorescently labeled plasma membrane marker LTI6B-mRFP (Kurup et al. 2005) were also carried out. For this, the $p U B N-R F P-L T I 6 b$ plasmid was used (kindly provided by Dr. K. Schumaker, Heidelberg, Germany). The subcellular localization of the fusion proteins was determined by CLSM at $52 \mathrm{~h}$ after agroinfiltration in an Olympus FV1000 microscope. The excitation wavelength was $488 \mathrm{~nm}$ for GFP and $543 \mathrm{~nm}$ for RFP. The emission window was set at 500 to $530 \mathrm{~nm}$ for GFP and 570 to 670 for RFP.

\section{Yeast functional complementation.}

The cDNA sequences encoding either the 1-NRAMP6 or s-NRAMP6 protein were amplified by PCR using primers BglII_lNramp6 and SalI_l-Nramp6 (for l-Nramp6) or the BglII_sNramp6 and SalI_s-Nramp6 primers (for $s$-Nramp6). The PCR products were cloned into the pGEM T-Easy vector and then inserted into the pWS93 yeast vector for N-terminal HAtagged expression under the control of the $A D H 1$ promoter.
Saccharomyces cerevisiae strains were grown in yeastpeptone-dextrose (YPD) medium (yeast extract at $10 \mathrm{~g} /$ liter, peptone at $20 \mathrm{~g} / \mathrm{liter}$, and dextrose at $20 \mathrm{~g} / \mathrm{liter}$ ) or, when carrying plasmids, in synthetic minimal medium lacking uracil (Adams et al. 1997). Growth assays on agar plates were performed as described previously (Posas et al. 1995). In some experiments, low-YPD medium (yeast extract at $2.5 \mathrm{~g} /$ liter, peptone at $5 \mathrm{~g} / \mathrm{liter}$, and dextrose at $20 \mathrm{~g} /$ liter) was employed. Wild-type $S$. cerevisiae strains BY4741 and its isogenic derivatives smf1::kanMX4, smf $2:: k a n M X 4$, and fet $3:: k a n M X 4$ were obtained from the EUROSCARF collection. Strain ASC64 (BY4741 fet3::kanMX4 fet $4:: n a t 1$ ) was constructed by transformation of the $f e t 3$ strain with a 1.25-kbp fet4::natl disruption cassette amplified from plasmid pAG25 (Goldstein and McCusker 1999) with oligonucleotides FET4_5'_NAT and FET4_3'_NAT.

\section{Western blot analysis of yeast protein extracts.}

For the preparation of yeast protein extracts, $10 \mathrm{ml}$ of cell culture was collected by centrifugation $(4 \mathrm{~min}, 546 \times g)$ and washed with cold double-distilled water, and the pellet was stored at $-80^{\circ} \mathrm{C}$. Cells were resuspended in $300 \mu \mathrm{l}$ of disruption buffer, and fractionation of the extract to separate soluble from precipitable (membrane-bound) material was performed as described by Pérez-Valle and associates (2007). Pellets were resuspended in $100 \mu \mathrm{l}$ of loading buffer, heated at $95^{\circ} \mathrm{C}(3 \mathrm{~min})$, and loaded onto sodium dodecyl sulfate polyacrylamide gel electrophoresis gels $(20 \mu \mathrm{l}$ of each sample). Proteins were transferred onto polyvinylidene difluoride membranes (Immobilon-P; Millipore). The NRAMP6 proteins were immunodetected using a mouse monoclonal anti-HA antibody (12CA4; 1:1,000 dilution; Roche). The secondary antimouse immunoglobulin G-horseradish peroxidase (GE Healthcare) was used at a 1:20,000 dilution, and immunoreactive proteins were visualized with the ECL Select kit (GE Healthcare).

\section{Analysis of the nramp6 mutant.}

Genotyping of nramp6 plants was carried out by PCR on genomic DNA using OsNramp6-specific primers (HY22_F and HY22_R) in combination with a T-DNA-specific primer (HY22_I, located at the left border of the T-DNA). Homozygous and azygous plants were identified. Nramp6 expression was examined in mutant plants by RT-qPCR, as described above. The T-DNA copy number was evaluated by RT-qPCR using the sucrose phosphate synthase gene as the endogenous reference gene (Yang et al. 2005).

\section{Blast resistance assay.}

The fungus M. oryzae (strain Guy-11) was grown in corn meal agar medium $(9-\mathrm{cm}$ plates containing chloramphenicol at $30 \mathrm{mg} /$ liter) for 2 weeks at $28^{\circ} \mathrm{C}$ under a photoperiod of $16 \mathrm{~h}$ of light and $8 \mathrm{~h}$ of darkness. M. oryzae spores were prepared as previously described (Campo et al. 2013). Infections were carried out by spraying the leaves of rice plants at the three-leaf stage (minimum 8 to 10 plants/experiment), whether soil-grown (susceptible Nipponbare and Hwayoung) or hydroponically grown (susceptible Nipponbare), with a spore suspension $\left(10^{5}\right.$ spores $\left./ \mathrm{ml}\right)$. Development of disease symptoms with time was followed. The percentage of leaf area affected by blast lesions was determined by using the APS Assess 2.0 program, typically at $7 \mathrm{dpi}$. Quantification of fungal DNA on infected leaves was carried out by qPCR using specific primers for $M$. oryzae 28S DNA (Mo28S), and the Ubiquitinl (Os06g46770) gene as the internal control.

\section{Metal content determination.}

Rice tissues were lyophilized, and 80 to $100 \mathrm{mg}$ (dry weight) was digested in $2 \mathrm{ml}$ of $65 \% \mathrm{HNO}_{3}$ and $1 \mathrm{ml}$ of $\mathrm{H}_{2} \mathrm{O}_{2}$ overnight at $100^{\circ} \mathrm{C}$. Samples were diluted in deionized water and the $\mathrm{Mn}$ and $\mathrm{Fe}$ content was measured by ICP-OES at the Scientific and Technological 
Center from the University of Barcelona. Concentrations were calculated by comparison with metal standards. At least three biological replicates for each condition tested were assayed.

\section{ACKNOWLEDGMENTS}

We thank J. M. Pardo and K. Schumaker for the pUBN-RFP-LTI6b plasma membrane marker, R. Camargo and J. Civera for assistance in parts of this work, and B. Oliva for helpful advice on structure modeling. This project was funded by the Ministry of Economy and Competitiveness (MINECO) and the European Regional Development's funds (BIO201232838 and BIO2015-67212 to B. San Segundo and BFU2014-54591-C2-1-P to J. Ariño). J. Ariño is recipient of a 2014SGR-4 grant from the Generalitat de Catalunya. We acknowledge the support of the MINECO for the "Centro de Excelencia Severo Ochoa 2016-2019” award SEV-2015-0533.

\section{LITERATURE CITED}

Adams, A., Gottschling, D., Kaiser, C., and Stearns, T. 1997. Methods in Yeast Genetics: A Cold Spring Harbor Laboratory Course Manual. Cold Spring Harbor Laboratory Press, Plainview, NY.

Agrawal, G. K., Rakwal, R., Jwa, N. S., and Agrawal, V. P. 2001. Signalling molecules and blast pathogen attack activates rice OsPRIa and OsPRIb genes: A model illustrating components participating during defence/stress response. Plant Physiol. Biochem. 39:1095-1103.

Alonso, J. M., Hirayama, T., Roman, G., Nourizadeh, S., and Ecker, J. R. 1999. EIN2, a bifunctional transducer of ethylene and stress responses in Arabidopsis. Science 284:2148-2152.

Anguelova-Merhar, V., Van der Westhuizen, A., and Pretorius, Z. 2002. Intercellular chitinase and peroxidase activities associated with resistance conferred by gene $L r 35$ to leaf rust of wheat. J. Plant Physiol. 159:1259-1261.

Belouchi, A., Kwan, T., and Gros, P. 1997. Cloning and characterization of the OsNramp family from Oryza sativa, a new family of membrane proteins possibly implicated in the transport of metal ions. Plant Mol. Biol. 33:1085-1092.

Blackwell, J. M., Searle, S., Mohamed, H., and White, J. K. 2003. Divalent cation transport and susceptibility to infectious and autoimmune disease: Continuation of the Ity/Lsh/Bcg/Nrampl/Slcllal gene story. Immunol. Lett. 85:197-203.

Brugiolo, M., Herzel, L., and Neugebauer, K. M. 2013. Counting on cotranscriptional splicing. F1000Prime Rep. 5:9.

Cailliatte, R., Lapeyre, B., Briat, J.-F., Mari, S., and Curie, C. 2009. The NRAMP6 metal transporter contributes to cadmium toxicity. Biochem. J. 422:217-228.

Cailliatte, R., Schikora, A., Briat, J.-F., Mari, S., and Curie, C. 2010. Highaffinity manganese uptake by the metal transporter NRAMP1 is essential for Arabidopsis growth in low manganese conditions. Plant Cell 22:904-917.

Campo, S., Peris-Peris, C., Siré, C., Moreno, A. B., Donaire, L., Zytnicki, M., Notredame, C., Llave, C., and San Segundo, B. 2013. Identification of a novel microRNA (miRNA) from rice that targets an alternatively spliced transcript of the Nramp6 (Natural resistance-associated macrophage protein 6) gene involved in pathogen resistance. New Phytol. 199:212-227.

Campos-Soriano, L., García-Martínez, J., and San Segundo, B. 2012. The arbuscular mycorrhizal symbiosis promotes the systemic induction of regulatory defence-related genes in rice leaves and confers resistance to pathogen infection. Mol. Plant Pathol. 13:579-592.

Cellier, M., Belouchi, A., and Gros, P. 1996. Resistance to intracellular infections: Comparative genomic analysis of Nramp. Trends Genet. 12:201-204.

Cellier, M., Privé, G., Belouchi, A., Kwan, T., Rodrigues, V., Chia, W., and Gros, P. 1995. Nramp defines a family of membrane proteins. Proc. Natl. Acad. Sci. U.S.A. 92:10089-10093.

Curie, C., Alonso, J. M., Le Jean, M., Ecker, J. R., and Briat, J.-F. 2000. Involvement of NRAMP1 from Arabidopsis thaliana in iron transport. Biochem. J. 347:749-755.

Curie, C., and Briat, J.-F. 2003. Iron transport and signaling in plants. Annu. Rev. Plant Biol. 54:183-206.

Dean, R., Van Kan, J. A. L., Pretorius, Z. A., Hammond-Kosack, K. E., Di Pietro, A., Spanu, P. D., Rudd, J. J., Dickman, M., Kahmann, R., Ellis, J., and Foster, G. D. 2012. The top 10 fungal pathogens in molecular plant pathology. Mol. Plant Pathol. 13:414-430.

de Carvalho Victoria, F., Bervald, C. M. P., da Maia, L. C., de Sousa, R. O., Panaud, O., and de Oliveira, A. C. 2012. Phylogenetic relationships and selective pressure on gene families related to iron homeostasis in land plants. Genome 55:883-900.

Delaunois, B., Jeandet, P., Clément, C., Baillieul, F., Dorey, S., and Cordelier, S. 2014. Uncovering plant-pathogen crosstalk through apoplastic proteomic studies. Front. Plant Sci. 5:249.
Dellagi, A., Segond, D., Rigault, M., Fagard, M., Simon, C., Saindrenan, P., and Expert, D. 2009. Microbial siderophores exert a subtle role in Arabidopsis during infection by manipulating the immune response and the iron status. Plant Physiol. 150:1687-1696.

Doehlemann, G., and Hemetsberger, C. 2013. Apoplastic immunity and its suppression by filamentous plant pathogens. New Phytol. 198:1001-1016.

Ehrnstorfer, I. A., Geertsma, E. R., Pardon, E., Steyaert, J., and Dutzler, R. 2014. Crystal structure of a SLC11 (NRAMP) transporter reveals the basis for transition-metal ion transport. Nat. Struct. Mol. Biol. 21:990-996.

Expert, D., Franza, T., and Dellagi, A. 2012. Iron in plant-pathogen interactions. Pages 7-39 in: Molecular Aspects of Iron Metabolism in Pathogenic and Symbiotic Plant-Microbe Associations. D. Expert and M. R. O'Brian, ed. Springer, Berlin Heidelberg.

Fones, H., and Preston, G. M. 2012. Reactive oxygen and oxidative stress tolerance in plant pathogenic Pseudomonas. FEMS Microbiol. Lett. 327:1-8.

Goldstein, A. L., and McCusker, J. H. 1999. Three new dominant drug resistance cassettes for gene disruption in Saccharomyces cerevisiae. Yeast 15:1541-1553.

Gross, J., Stein, R. J., Fett-Neto, A. G., and Fett, J. P. 2003. Iron homeostasis related genes in rice. Genet. Mol. Biol. 26:477-497.

Guerinot, M. L. 2000. The ZIP family of metal transporters. Biochim. Biophys. Acta 1465:190-198.

Gupta, S. K., Rai, A. K., Kanwar, S. S., Chand, D., Singh, N. K., and Sharma, T. R. 2012. The single functional blast resistance gene Pi54 activates a complex defence mechanism in rice. J. Exp. Bot. 63:757-772.

Hall, J. L., and Williams, L. E. 2003. Transition metal transporters in plants. J. Exp. Bot. 54:2601-2613.

Hirokawa, T., Boon-Chieng, S., and Mitaku, S. 1998. SOSUI: Classification and secondary structure prediction system for membrane proteins. Bioinformatics 14:378-379.

Hof, C., Eisfeld, K., Antelo, L., Foster, A. J., and Anke, H. 2009. Siderophore synthesis in Magnaporthe grisea is essential for vegetative growth, conidiation and resistance to oxidative stress. Fungal Genet. Biol. 46:321-332.

Hof, C., Eisfeld, K., Welzel, K., Antelo, L., Foster, A. J., and Anke, H. 2007. Ferricrocin synthesis in Magnaporthe grisea and its role in pathogenicity in rice. Mol. Plant Pathol. 8:163-172.

Hood, M. I., and Skaar, E. P. 2012. Nutritional immunity: Transition metals at the pathogen-host interface. Nat. Rev. Microbiol. 10:525-537.

Huot, B., Yao, J., Montgomery, B. L., and He, S. Y. 2014. Growth-defense tradeoffs in plants: A balancing act to optimize fitness. Mol. Plant 7:1267-1287.

Inoue, H., Kobayashi, T., Nozoye, T., Takahashi, M., Kakei, Y., Suzuki, K., Nakazono, M., Nakanishi, H., Mori, S., and Nishizawa, N. K. 2009. Rice OsYSL15 is an iron-regulated iron(III)-deoxymugineic acid transporter expressed in the roots and is essential for iron uptake in early growth of the seedlings. J. Biol. Chem. 284:3470-3479.

Ishimaru, Y., Masuda, H., Bashir, K., Inoue, H., Tsukamoto, T., Takahashi, M., Nakanishi, H., Aoki, N., Hirose, T., Ohsugi, R., and Nishizawa, N. K. 2010. Rice metal-nicotianamine transporter, OsYSL2, is required for the long-distance transport of iron and manganese. Plant J. 62:379-390.

Ishimaru, Y., Suzuki, M., Tsukamoto, T., Suzuki, K., Nakazono, M., Kobayashi, T., Wada, Y., Watanabe, S., Matsuhashi, S., Takahashi, M., Nakanishi, H., Mori, S., and Nishizawa, N. K. 2006. Rice plants take up iron as an Fe3+-phytosiderophore and as Fe2+. Plant J. 45:335-346.

Ishimaru, Y., Takahashi, R., Bashir, K., Shimo, H., Senoura, T., Sugimoto, K., Ono, K., Yano, M., Ishikawa, S., Arao, T., Nakanishi, H., and Nishizawa, N. K. 2012. Characterizing the role of rice NRAMP5 in manganese, iron and cadmium transport. Sci. Rep. 2:286-293.

Jun, L., Saiki, R., Tatsumi, K., Nakagawa, T., and Kawamukai, M. 2004. Identification and subcellular localization of two solanesyl diphosphate synthases from Arabidopsis thaliana. Plant Cell Physiol. 45:1882-1888.

Kankanala, P., Czymmek, K., and Valent, B. 2007. Roles for rice membrane dynamics and plasmodesmata during biotrophic invasion by the blast fungus. Plant Cell 19:706-724.

Karimi, M., Inzé, D., and Depicker, A. 2002. GATEWAY ${ }^{\mathrm{TM}}$ vectors for Agrobacterium-mediated plant transformation. Trends Plant Sci. 7:193-195.

Kieu, N. P., Aznar, A., Segond, D., Rigault, M., Simond-Côte, E., Kunz, C., Soulie, M.-C., Expert, D., and Dellagi, A. 2012. Iron deficiency affects plant defence responses and confers resistance to Dickeya dadantii and Botrytis cinerea. Mol. Plant Pathol. 13:816-827.

Kim, S. G., Wang, Y., Lee, K. H., Park, Z.-Y., Park, J., Wu, J., Kwon, S. J., Lee, Y.-H., Agrawal, G. K., Rakwal, R., Kim, S. T., and Kang, K. Y. 2013. In-depth insight into in vivo apoplastic secretome of riceMagnaporthe oryzae interaction. J. Proteomics 78:58-71.

Kobayashi, T., and Nishizawa, N. K. 2012. Iron uptake, translocation, and regulation in higher plants. Annu. Rev. Plant Biol. 63:131-152.

Kurup, S., Runions, J., Köhler, U., Laplaze, L., Hodge, S., and Haseloff, J. 2005. Marking cell lineages in living tissues. Plant J. 42:444-453. 
Lanquar, V., Lelièvre, F., Barbier-Brygoo, H., and Thomine, S. 2004 Regulation and function of AtNRAMP4 metal transporter protein. Soil Sci. Plant Nutr. 50:1141-1150.

Lanquar, V., Lelièvre, F., Bolte, S., Hamès, C., Alcon, C., Neumann, D., Vansuyt, G., Curie, C., Schröder, A., Krämer, U., Barbier-Brygoo, H., and Thomine, S. 2005. Mobilization of vacuolar iron by AtNRAMP3 and AtNRAMP4 is essential for seed germination on low iron. EMBO J. 24:4041-4051.

Lanquar, V., Ramos, M. S., Lelièvre, F., Barbier-Brygoo, H., KriegerLiszkay, A., Krämer, U., and Thomine, S. 2010. Export of vacuolar manganese by AtNRAMP3 and AtNRAMP4 is required for optimal photosynthesis and growth under manganese deficiency. Plant Physiol. 152:1986-1999.

Lemanceau, P., Expert, D., Gaymard, F., Bakker, P. A. H. M., and Briat, J. F. 2009. Role of iron in plant-microbe interactions. Pages 491-549 in: Advances in Botanical Research. L. C. Van Loon, ed. Elsevier, Amsterdam.

Li, J.-Y., Liu, J., Dong, D., Jia, X., McCouch, S. R., and Kochian, L. V. 2014. Natural variation underlies alterations in Nramp aluminum transporter (NRAT1) expression and function that play a key role in rice aluminum tolerance. Proc. Natl. Acad. Sci. U.S.A. 111:6503-6508.

Liu, G., Greenshields, D. L., Sammynaiken, R., Hirji, R. N., Selvaraj, G., and Wei, Y. 2007. Targeted alterations in iron homeostasis underlie plant defense responses. J. Cell Sci. 120:596-605.

Liu, J., Chen, X., Liang, X., Zhou, X., Yang, F., Liu, J., He, S. Y., and Guo, Z. 2016. Alternative splicing of rice WRKY62 and WRKY76 transcription factor genes in pathogen defense. Plant Physiol. 171:1427-1442.

Nelson, N. 1999. Metal ion transporters and homeostasis. EMBO J. 18: 4361-4371.

Nevo, Y., and Nelson, N. 2006. The NRAMP family of metal-ion transporters. Biochim. Biophys. Acta 1763:609-620.

Pérez-Valle, J., Jenkins, H., Merchan, S., Montiel, V., Ramos, J., Sharma, S. Serrano, R., and Yenush, L. 2007. Key role for intracellular K+ and protein kinases Sat4/Hal4 and Hal5 in the plasma membrane stabilization of yeast nutrient transporters. Mol. Cell. Biol. 27:5725-5736.

Pettersen, E. F., Goddard, T. D., Huang, C. C., Couch, G. S., Greenblatt, D. M., Meng, E. C., and Ferrin, T. E. 2004. UCSF Chimera-A visualization system for exploratory research and analysis. J. Comput. Chem. 25: 1605-1612.

Portnoy, M. E., Liu, X. F., and Culotta, V. C. 2000. Saccharomyces cerevisiae expresses three functionally distinct homologues of the nramp family of metal transporters. Mol. Cell. Biol. 20:7893-7902.

Posas, F., Camps, M., and Ariño, J. 1995. The PPZ protein phosphatases are important determinants of salt tolerance in yeast cells. J. Biol. Chem. 270:13036-13041.

Pottier, M., Oomen, R., Picco, C., Giraudat, J., Scholz-Starke, J., Richaud, P., Carpaneto, A., and Thomine, S. 2015. Identification of mutations allowing Natural Resistance Associated Macrophage Proteins (NRAMP) to discriminate against cadmium. Plant J. 83:625-637.

Puig, S., and Peñarrubia, L. 2009. Placing metal micronutrients in context: Transport and distribution in plants. Curr. Opin. Plant Biol. 12:299-306.

Roy, A., Kucukural, A., and Zhang, Y. 2010. I-TASSER: A unified platform for automated protein structure and function prediction. Nat. Protoc. 5: 725-738.

Saitou, N., and Nei, M. 1987. The neighbor-joining method: A new method for reconstructing phylogenetic trees. Mol. Biol. Evol. 4:406-425.

Sasaki, A., Yamaji, N., Yokosho, K., and Ma, J. F. 2012. Nramp5 is a major transporter responsible for manganese and cadmium uptake in rice. Plant Cell 24:2155-2167.

Schwach, F., Vaistij, F. E., Jones, L., and Baulcombe, D. C. 2005. An RNAdependent RNA polymerase prevents meristem invasion by potato virus $\mathrm{X}$ and is required for the activity but not the production of a systemic silencing signal. Plant Physiol. 138:1842-1852.

Segond, D., Dellagi, A., Lanquar, V., Rigault, M., Patrit, O., Thomine, S., and Expert, D. 2009. NRAMP genes function in Arabidopsis thaliana resistance to Erwinia chrysanthemi infection. Plant J. 58:195-207.

Sippl, M. J. 1993. Recognition of errors in three-dimensional structures of proteins. Proteins 17:355-362.

Takahashi, R., Ishimaru, Y., Senoura, T., Shimo, H., Ishikawa, S., Arao, T., Nakanishi, H., and Nishizawa, N. K. 2011. The OsNRAMP1 iron transporter is involved in $\mathrm{Cd}$ accumulation in rice. J. Exp. Bot. 62: 4843-4850.

Tamura, K., Dudley, J., Nei, M., and Kumar, S. 2007. MEGA4: Molecular Evolutionary Genetics Analysis (MEGA) software version 4.0. Mol. Biol. Evol. 24:1596-1599.

Tejada-Jiménez, M., Castro-Rodríguez, R., Kryvoruchko, I., Lucas, M. M. Udvardi, M., Imperial, J., and González-Guerrero, M. 2015. Medicago truncatula natural resistance-associated macrophage Protein 1 is required for iron uptake by rhizobia-infected nodule cells. Plant Physiol. 168: 258-272.

Thomine, S., Lelièvre, F., Debarbieux, E., Schroeder, J. I., and BarbierBrygoo, H. 2003. AtNRAMP3, a multispecific vacuolar metal transporter involved in plant responses to iron deficiency. Plant J. 34:685-695.

Thomine, S., and Vert, G. 2013. Iron transport in plants: Better be safe than sorry. Curr. Opin. Plant Biol. 16:322-327.

Thomine, S., Wang, R., Ward, J. M., Crawford, N. M., and Schroeder, J. I. 2000. Cadmium and iron transport by members of a plant metal transporter family in Arabidopsis with homology to Nramp genes. Proc. Natl. Acad. Sci. U.S.A. 97:4991-4996.

Tiwari, M., Sharma, D., Dwivedi, S., Singh, M., Tripathi, R. D., and Trivedi, P. K. 2014. Expression in Arabidopsis and cellular localization reveal involvement of rice NRAMP, OsNRAMP1, in arsenic transport and tolerance. Plant Cell Environ. 37:140-152.

Torres, M. A., Jones, J. D., and Dangl, J. L. 2006. Reactive oxygen species signaling in response to pathogens. Plant Physiol. 141:373-378.

Vidal, S. M., Malo, D., Vogan, K., Skamene, E., and Gros, P. 1993. Natural resistance to infection with intracellular parasites: Isolation of a candidate for $\mathrm{Bcg}$. Cell 73:469-485.

Wally, O., Jayaraj, J., and Punja, Z. 2009. Comparative resistance to foliar fungal pathogens in transgenic carrot plants expressing genes encoding for chitinase, $\beta$-1,3-glucanase and peroxidise. Eur. J. Plant Pathol. 123:331-342.

Wiederstein, M., and Sippl, M. J. 2007. ProSA-web: Interactive web service for the recognition of errors in three-dimensional structures of proteins. Nucleic Acids Res. 35:W407-W410.

Williams, L. E., and Pittman, J. K. 2010. Dissecting pathways involved in manganese homeostasis and stress in higher plant cells. Pages 95-117 in Cell Biology of Metals and Nutrients. R. Hell and R. Mendel, eds. Springer, Berlin, Heidelberg.

Wilson, R. A., Fernandez, J., Quispe, C. F., Gradnigo, J., Seng, A., Moriyama, E., and Wright, J. D. 2012. Towards defining nutrient conditions encountered by the rice blast fungus during host infection. PLoS One 7:e47392.

Wilson, R. A., and Talbot, N. J. 2009. Under pressure: Investigating the biology of plant infection by Magnaporthe oryzae. Nat. Rev. Microbiol. 7:185-195

Wu, D., Yamaji, N., Yamane, M., Kashino-Fujii, M., Sato, K., and Feng Ma, J. 2016. The HvNramp5 transporter mediates uptake of cadmium and manganese, but not iron. Plant Physiol. 172:1899-1910.

Xia, J., Yamaji, N., Kasai, T., and Ma, J. F. 2010. Plasma membranelocalized transporter for aluminum in rice. Proc. Natl. Acad. Sci. U.S.A 107:18381-18385.

Xiao, H., Yin, L., Xu, X., Li, T., and Han, Z. 2008. The iron-regulated transporter, MbNRAMP1, isolated from Malus baccata is involved in $\mathrm{Fe}$ Mn and Cd trafficking. Ann. Bot. (Lond.) 102:881-889.

Yamaji, N., Sasaki, A., Xia, J. X., Yokosho, K., and Ma, J. F. 2013. A nodebased switch for preferential distribution of manganese in rice. Nat. Commun. 4:2442.

Yang, L., Ding, J., Zhang, C., Jia, J., Weng, H., Liu, W., and Zhang, D. 2005. Estimating the copy number of transgenes in transformed rice by real-time quantitative PCR. Plant Cell Rep. 23:759-763.

Yang, M., Zhang, W., Dong, H., Zhang, Y., Lv, K., Wang, D., and Lian, X 2013. OsNRAMP3 is a vascular bundles-specific manganese transporter that is responsible for manganese distribution in rice. PLoS One 8: e83990.

Young, S. A., Guo, A., Guikema, J. A., White, F. F., and Leach, J. E. 1995. Rice cationic peroxidase accumulates in xylem vessels during incompatible interactions with Xanthomonas oryzae pv. oryzae. Plant Physiol. 107:1333-1341.

Zhou, X., and Yang, Y. 2004. Differential expression of rice Nramp genes in response to pathogen infection, defense signal molecules and metal ions. Physiol. Mol. Plant Pathol. 65:235-243.

\section{AUTHOR-RECOMMENDED INTERNET RESOURCES}

Boxshade program: http://www.ch.embnet.org/software/BOX_doc.html ClustalW database: http://clustalw.ddbj.nig.ac.jp MEGA4 software: http://megasoftware.net POSTECH RISD website: http://cbi.khu.ac.kr ProSA program: https://prosa.services.came.sbg.ac.at RCSB Protein Data Bank: http://www.rcsb.org Rice Genome Annotation Project database: http://rice.plantbiology.msu.edu Rice Genome Resource Center webpage: http://www.rgrc.dna.affrc.go.jp SOSUI prediction tool: http://harrier.nagahama-i-bio.ac.jp/sosui UCSF Chimera package: http://www.cgl.ucsf.edu/chimera 